\title{
The EDGE-CALIFA Survey: Variations in the Molecular Gas Depletion Time in Local Galaxies
}

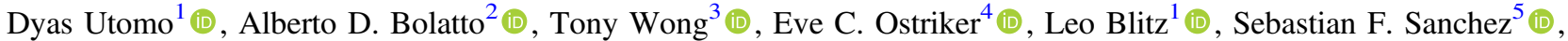

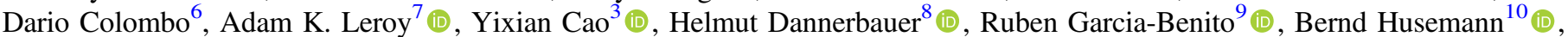 \\ Veselina Kalinova ${ }^{6}$, Rebecca C. Levy ${ }^{2}$, Damian Mast ${ }^{11}{ }^{1}$, Erik Rosolowsky $^{12}{ }^{(1)}$, and Stuart N. Vogel ${ }^{2}$ \\ ${ }^{1}$ Department of Astronomy, University of California, Berkeley, CA 94704, USA; dyas@berkeley.edu \\ ${ }^{2}$ Department of Astronomy, University of Maryland, College Park, MD 20642, USA \\ ${ }^{3}$ Department of Astronomy, University of Illinois, Urbana, IL 61801, USA \\ ${ }^{4}$ Department of Astrophysical Sciences, Princeton University, Princeton, NJ 08544, USA \\ ${ }^{5}$ Instituto de Astronomía, Universidad Nacional Autónoma de México, A.P. 70-264, 04510 México, D.F., Mexico \\ ${ }^{6}$ Max Planck Institute for Radio Astronomy, Auf dem Hügel 69, D-53121 Bonn, Germany \\ ${ }^{7}$ Department of Astronomy, The Ohio State University, Columbus, OH 43210, USA \\ ${ }^{8}$ Instituto de Astrofísica de Canarias, E-38205 La Laguna, Tenerife, Spain \\ ${ }^{9}$ Instituto de Astrofísica de Andalucía, CSIC, E-18008 Granada, Spain \\ ${ }^{10}$ Max Planck Institute für Astronomie, Königstuhl 17, D-69117 Heidelberg, Germany \\ ${ }_{11}^{11}$ Observatorio Astronómico de Córdoba, 5000 Córdoba, Córdoba, Argentina \\ ${ }^{12}$ Department of Physics, University of Alberta, Edmonton, T6G 2E1, Canada \\ Received 2017 April 8; revised 2017 August 22; accepted 2017 August 24; published 2017 October 26
}

\begin{abstract}
We present results from the EDGE survey, a spatially resolved $\mathrm{CO}(1-0)$ follow-up to CALIFA, an optical Integral Field Unit survey of local galaxies. By combining the data products of EDGE and CALIFA, we study the variation in molecular gas depletion time $\left(\tau_{\text {dep }}\right)$ on kiloparsec scales in 52 galaxies. We divide each galaxy into two parts: the center, defined as the region within $0.1 R_{25}$, and the disk, defined as the region between 0.1 and $0.7 R_{25}$. We find that 14 galaxies show a shorter $\tau_{\text {dep }}(\sim 1 \mathrm{Gyr})$ in the center relative to that in the disk $\left(\tau_{\text {dep }}\right.$ $\sim 2.4 \mathrm{Gyr}$ ), which means the central region in those galaxies is more efficient at forming stars per unit molecular gas mass. This finding implies that the centers with shorter $\tau_{\text {dep }}$ resemble the intermediate regime between galactic disks and starburst galaxies. Furthermore, the central drop in $\tau_{\text {dep }}$ is correlated with a central increase in the stellar surface density, suggesting that a shorter $\tau_{\text {dep }}$ is associated with molecular gas compression by the stellar gravitational potential. We argue that varying the CO-to- $\mathrm{H}_{2}$ conversion factor only exaggerates the central drop of $\tau_{\text {dep. }}$.
\end{abstract}

Key words: galaxies: star formation - galaxies: structure - ISM: abundances - ISM: molecules

\section{Introduction}

Galactic stellar masses grow through a combination of mergers and the formation of stars from their gas reservoir over cosmic time. Therefore, the star-formation rate (SFR) is an important factor in driving galaxy evolution (e.g., Kennicutt 1998a; McKee \& Ostriker 2007; Kennicutt \& Evans 2012). In general, star formation involves two processes: (1) the conversion of diffuse, atomic gas into molecular gas in well-shielded regions of high density and (2) the dynamical collapse of self-gravitating regions within the molecular component to form stars. In galactic regions with low mean gas volume and low surface density, local gas compression by spiral arms or self-gravity may be needed for molecules to form, whereas in galactic regions of high mean gas volume and surface density, most of the gas is already molecular (e.g., in M51; Schinnerer et al. 2013). In this paper, we focus on the second part of the star-formation processes; specifically, we study how the relation between molecular gas and SFR changes between the galactic centers and the disks.

In a simple-minded picture, stars form from the gas that contracts under its self-gravity. Naively, one would expect that the relevant timescale of this process is the free-fall time $\left(\tau_{\mathrm{ff}}\right)$ of the total gas (atomic and molecular), which is inversely proportional to the square-root of gas volume density $\left(\rho_{\text {gas }}^{-0.5}\right)$. The implication of this simple scenario is that SFR relates to the amount of gas as $\rho_{\mathrm{SFR}} \propto \rho_{\mathrm{gas}} / \tau_{\mathrm{ff}} \propto \rho_{\mathrm{gas}}^{1.5}{ }^{13}$ In general, the relation between SFR and total gas density is called the Kennicutt-Schmidt (KS) relation, after the seminal papers by Schmidt (1959) and Kennicutt (1998b). ${ }^{14}$

Observations in the local universe show that stars form in molecular clouds, so we expect that SFR correlates better with the amount of molecular gas, rather than the total amount of atomic plus molecular gas (e.g., Wong \& Blitz 2002; Kennicutt et al. 2007; Bigiel et al. 2008). Even though the molecular phase may itself not be necessary to form stars (Glover \& Clark 2012), molecular gas that forms under the high-density conditions are also favorable to gravitational collapse, thus giving rise to a strong KS relation (Krumholz et al. 2011). For simplicity, in this paper, we refer to the relationship between SFR and molecular gas surface densities as the $\mathrm{KS}$ relation.

Resolved studies of nearby galaxies found that the correlation between SFR and molecular gas surface densities is

\footnotetext{
13 The other timescales that are often used in the literature are the orbital time $\Omega^{-1}$ (e.g., Elmegreen 1997; Silk 1997), where $\Omega$ is the angular speed of the disk, and the vertical time $H / \sigma$ (Ostriker et al. 2010; Ostriker \& Shetty 2011), where $H$ and $\sigma$ are the thickness and velocity dispersion of the gas.

${ }^{14}$ Actually, Schmidt (1959) proposed $\rho_{\mathrm{SFR}} \propto \rho_{\text {gas }}^{2}$ and Kennicutt (1998b) found $\Sigma_{\text {SFR }} \propto \Sigma_{\text {gas }}^{1.4}$, where $\Sigma$ is the surface density. Since $\Sigma$ is the integration of $\rho$ along the projected disk thickness, the translation from $\rho_{\mathrm{SFR}}$ to $\Sigma_{\mathrm{SFR}}$ depends on the scale height of the ISM.
} 
approximately linear ${ }^{15}$ in galaxy disks, with $\Sigma_{\mathrm{SFR}} \propto \Sigma_{\mathrm{mol}}$ on kiloparsec (kpc) scales for surface densities $\Sigma_{\mathrm{H} 2} \gtrsim 3 M_{\odot} \mathrm{pc}^{-2}$ over a wide range of local environments (e.g., Bigiel et al. 2008; Leroy et al. 2008). Furthermore, in nearby galaxies, the near-linear molecular KS relation extends to the low-metallicity regime $\left(Z / Z_{\odot} \approx 0.2\right.$; Bolatto et al. 2011; Jameson et al. 2016) and to the outer part of galaxies, where the gas surface density is low and atomic dominated (Schruba et al. 2011). A possible reason for this widespread relationship is that the properties of molecular clouds are similar from one galaxy and region to another (Bolatto et al. 2008), so that GMCs convert the molecular gas into stars at the same rate.

For most of the gas in normal galaxies, the linearity of the KS relation implies the molecular gas depletion time, defined as $\tau_{\text {dep }} \equiv \Sigma_{\text {mol }} / \Sigma_{\text {SFR }}$, is approximately constant, with a typical value of 2.2 Gyr in nearby galaxies (e.g., Bigiel et al. 2008; Leroy et al. 2008, 2013; Rahman et al. 2012). Loosely, we interpret $\tau_{\text {dep }}$ as the timescale to convert all of the molecular gas reservoir in a galaxy (or a given region within a galaxy) into stars at the current SFR. The fact that $\tau_{\text {dep }}$ is less than the Hubble time implies that galaxies need to replenish their molecular gas reservoirs through stellar feedback (e.g., supernovae, stellar winds, AGB stars, and planetary nebula), conversion from atomic to molecular gas, and accretion from the intergalactic medium or from satellite galaxies (e.g., Bauermeister et al. 2010; Genzel et al. 2010; Lilly et al. 2013). However, a direct observational signature of this accretion is still challenging.

Despite the current evidence toward the linearity of the KS relation, there are at least three regimes where this linearity breaks down: (1) in the ULIRGs and starburst galaxies, i.e., galaxies above the star-forming main sequence (e.g., Daddi et al. 2010; Genzel et al. 2010, 2015), (2) at a resolution finer than 500 pc (e.g., Schruba et al. 2010; Calzetti et al. 2012; Kruijssen \& Longmore 2014), and (3) in galactic centers (e.g., Jogee et al. 2005; Leroy et al. 2013). In addition, a trend of $\tau_{\text {dep }}$ with respect to stellar mass on a galaxy-by-galaxy basis was reported by Saintonge et al. (2011b) in a COLDGASS sample and Bolatto et al. (2017) in an EDGE sample.

The steeper-than-linear molecular KS relation in regions of very high molecular surface density has been interpreted as a result of higher molecular gas pressure (Ostriker \& Shetty 2011) and density (Krumholz et al. 2012). Higher pressure requires a higher SFR per unit molecular mass to offset enhanced turbulent dissipation and cooling, and higher density is associated with shorter dynamical times, which control gravitational contraction.

This paper is based on the combination of the $\mathrm{CO}$ data from the EDGE survey (Bolatto et al. 2017) and the optical Integral Field Unit (IFU) data from the CALIFA survey (Sánchez et al. 2012). In the first EDGE paper by Bolatto et al. (2017), we showed that the relation between $\Sigma_{\text {SFR }}$ and $\Sigma_{\text {mol }}$ is approximately linear, with a separation of $\tau_{\text {dep }}$ between high and low-mass galaxies. We extend that study in this paper by analyzing the variations of $\tau_{\text {dep }}$ between galactic centers and

\footnotetext{
15 There is a tension on the actual slope of the KS relation. For example, Kennicutt et al. (2007) derived a slope of 1.37 in M51, while Bigiel et al. (2008) derived a slope of 0.84 in the same galaxy. There are two possible reasons of this difference: (1) different treatments on the background radiation that is used as a tracer for SFR (Liu et al. 2011; Calzetti et al. 2012; a removal of background radiation leads to a steeper slope) and (2) different regions in M51 have different slopes of KS relation (Leroy et al. 2017), so that the derived slope depends on which regions have larger weight in the best-fit slope.
}

disks, with a goal to quantify and understand the cause of those variations and their implications in galaxy evolution.

This paper is organized as follows. Overviews of the EDGE and CALIFA data products and the sample selection are described in Sections 2 and 3, respectively. Then, in Section 4, we compare $\tau_{\text {dep }}$ in the centers relative to those in the disk. Specifically, we investigate whether the difference of $\tau_{\text {dep }}$ between the centers and the disks is due to SFR, molecular gas, or stellar surface density. In Section 5, we discuss the effect of the CO-to- $\mathrm{H}_{2}$ conversion factor, the connection between $\tau_{\text {dep }}$ and oxygen abundance, the size of molecular and stellar disks, and the possibility that the galactic center undergoes cycles of star formation. Lastly, we summarize our findings in Section 6. All logarithms in this paper are base 10 logarithms.

\section{Data Descriptions}

\subsection{The EDGE Survey}

The EDGE survey targets 126 galaxies in the $\mathrm{CO}(1-0)$ and ${ }^{13} \mathrm{CO}(1-0)$ lines using the CARMA observatory (Bock et al. 2006) in the D and E arrays from 2014 October until 2015 May. The observational details and data reductions of the EDGE survey are presented in Bolatto et al. (2017). Briefly, the EDGE samples are selected from the CALIFA Second Data Release (García-Benito et al. 2015) based on their fluxes in the WISE $22 \mu \mathrm{m}$ band (Wright et al. 2010). The raw data are reduced using the MIRIAD package (Sault et al. 1995) into data cubes (i.e., CO intensity in velocity and two-dimensional spaces) using an automated pipeline based on scripts developed for the STING galaxy survey (Rahman et al. 2012; Wong et al. 2013).

The beam size of each galaxy varies with a typical value of 4." 5, which corresponds to a median physical scale of about $1.5 \mathrm{kpc}$. This physical resolution is slightly larger than previous CO surveys, such as BIMA SONG ( 360 pc; Helfer et al. 2003), HERACLES ( 500 pc; Leroy et al. 2009), and STING (160 - 1250 pc; Rahman et al. 2012), because our sample covers farther median distance than those surveys. The pixel size is $2^{\prime \prime} \times 2^{\prime \prime}$. The velocity resolution is $10 \mathrm{~km} \mathrm{~s}^{-1}$ with a typical velocity range of $860 \mathrm{~km} \mathrm{~s}^{-1}$, thus, it covers out to the flat part of the rotation curve where $\mathrm{CO}$ is detected. The data cubes that provide an estimate of $1 \sigma_{\text {rms }}$ noise level at each pixel were also generated during the data reduction processes.

In order to separate signal from noise, we create masks through the following steps in IDL (code is available at https://github. com/tonywong94/idl_mommaps; Wong et al. 2013). First, we smooth the data into $9^{\prime \prime}$ resolutions with a Gaussian kernel. The aim of this smoothing is to reach a higher signal-to-noise ratio $(\mathrm{S} / \mathrm{N})$. Then, we search for contiguous regions, starting from a pixel that has $\mathrm{S} / \mathrm{N} \geqslant 3.5$ down to regions that have $\mathrm{S} / \mathrm{N}=2$. The aim of contiguous regions is to remove noise that has high $\mathrm{S} / \mathrm{N}$ by chance, but only localized into one to few pixels (e.g., Rosolowsky \& Leroy 2006). An additional padding of 2 pixels surrounding the $2 \times \mathrm{S} / \mathrm{N}$ contours are added into the mask to capture low level emission. Finally, we apply these masks to the data cubes in their original resolutions (4." 5 and $10 \mathrm{~km} \mathrm{~s}^{-1}$ ). We define these contiguous regions, including the padding, as masked regions.

The masked data cubes are integrated along the velocity axis to get the CO surface brightness maps (zeroth moment maps). Similarly, the uncertainties of the maps are taken by integrating the estimated noise along the velocity axis within the masked cubes. In the analyses, we use these uncertainty maps as the 

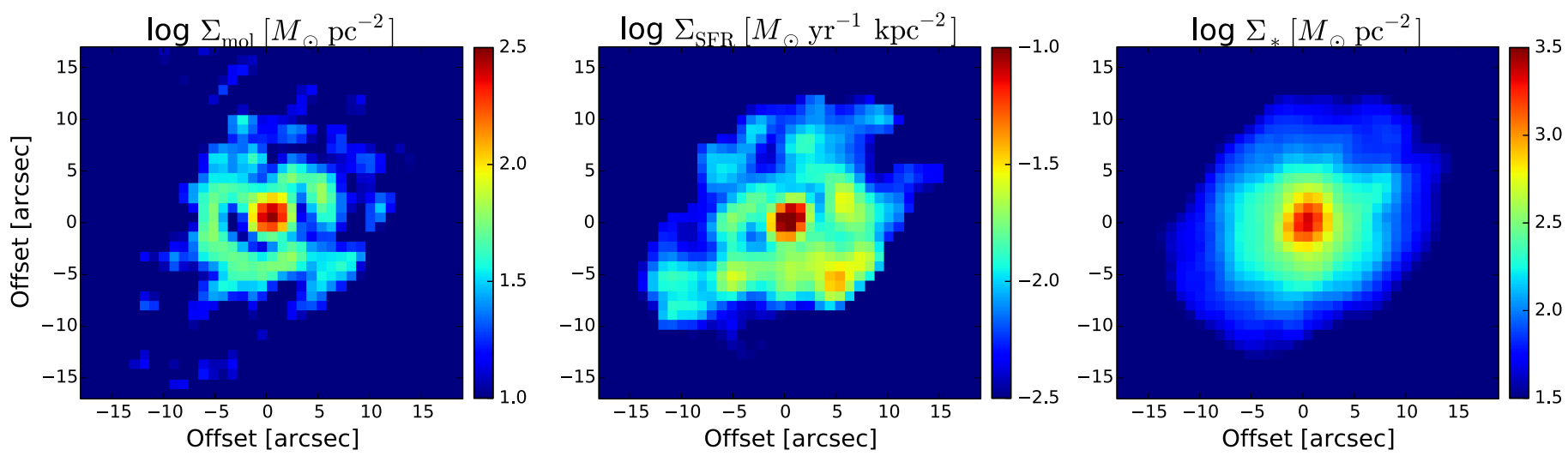

Figure 1. Example of the EDGE-CALIFA data products. From left to right: the maps of molecular gas, SFR, and stellar mass surface density of NGC 2253.

$1 \sigma_{\text {rms }}$ noise level. Note that not all masked $\mathrm{CO}$ surface brightness maps are higher than the $2 \sigma_{\text {rms }}$ level, therefore, we treat emissions below the $2 \sigma_{\text {rms }}$ level as non-detections, even though these emissions are located within the mask.

We convert the $\mathrm{CO}$ surface brightness and its uncertainty maps into molecular gas surface density $\left(\Sigma_{\mathrm{mol}}\right)$ maps by using a constant CO-to- $\mathrm{H}_{2}$ conversion factor $\left(\alpha_{\mathrm{CO}}\right)$ of $4.4 M_{\odot} \mathrm{pc}^{-2}$ $\left(\mathrm{K} \mathrm{km} \mathrm{s}^{-1} \mathrm{pc}^{2}\right)^{-1}$, including the mass contribution from helium. In general, $\alpha_{\mathrm{CO}}$ can vary as a function of metallicities and stellar surface densities (Bolatto et al. 2013). In our approach, we take a Galactic value of $\alpha_{\mathrm{CO}}$, and then, we consider how the variations of $\alpha_{\mathrm{CO}}$ affect our results in Section 5.1. Note that any surface density measurement has been corrected (deprojected) from inclination ( $i$ ) by using a correction factor of $\cos (i)$. An example of the map of $\Sigma_{\mathrm{mol}}$ is shown as the left panel of Figure 1.

\subsection{The CALIFA Survey}

CALIFA is an optical IFU survey of $\sim 600$ local galaxies at the redshift range of $0.005 \lesssim z \lesssim 0.03$ using the $3.5 \mathrm{~m}$ telescope at the Calar-alto observatory (Sánchez et al. 2012). The CALIFA samples are selected from the SDSS DR7 database (Abazajian et al. 2009) based on their diameter in the $r$-band ( $45^{\prime \prime}<D_{25}<80^{\prime \prime}$ ), so that they fit well within the IFU field of view of $1 ! 3$, or equivalently $\sim 2.5$ effective radius (Walcher et al. 2014), but statistically still represents the population of $z \sim 0$ galaxies in the color-magnitude diagram. In an IFU survey, we can get spatial and spectral information of an object, simultaneously. The spatial resolution of CALIFA is $\sim 2$ ! 5 (or $\sim \mathrm{kpc}$ scale) and the spectral range of CALIFA covers $3700-7000 \AA$, so that it captures the stellar absorption lines and the nebular emission lines.

We take the following additional steps to create homogeneous data sets between EDGE and CALIFA. (1) Recenter any offset in CALIFA data by using cross-correlation between CALIFA $V$ band and SDSS $g$-band images. In general, the offsets are about a few arcseconds and are not systematic. (2) Regrid the CALIFA data by using the MIRIAD task regrid, so that it has the same spatial coordinate as in the EDGE data with a common pixel size of $2^{\prime \prime} \times 2^{\prime \prime}$. In this process, we also degrade the resolution of CALIFA images to match the resolution of EDGE images by using the MIRIAD task convol. The total flux is conserved during those processes. (3) Blanking the CALIFA data that are contaminated by foreground stars and neighboring galaxies. (4) Separating signals from noise by blanking any pixels that have $S$ / $\mathrm{N}<2$, where we use the median absolute deviation of the
CALIFA image as an estimate of the noise. As in the EDGE data set, all surface densities derived from the CALIFA data set have been corrected by $\cos (i)$ to take into account the size deprojection due to inclination.

\subsubsection{The SFR Surface Density}

The post-processing results of CALIFA data (Pipe3D version 2.2 from Sánchez et al. 2016) provide the intensity maps of emission lines, such as $\mathrm{H} \alpha$ and $\mathrm{H} \beta$. To derive maps of the SFR surface density $\left(\Sigma_{\mathrm{SFR}}\right)$, first, we calculate the nebular extinction at $\mathrm{H} \alpha$ wavelength, $A_{\mathrm{H} \alpha}$, by utilizing the ratio of $\mathrm{H} \alpha$ and $\mathrm{H} \beta$ fluxes (Balmer decrement method; e.g., Domínguez et al. 2013) and compare it with its intrinsic value (zero extinction) of 2.86 (for case $\mathrm{B}$ recombination at temperature of $10^{4} \mathrm{~K}$ and electron density of $100 \mathrm{~cm}^{-3}$; Osterbrock 1989). In the process, we also use a Galactic extinction curve (Cardelli et al. 1989) with $R_{V}=3.1$. The result will be similar if we use Calzetti et al.'s (2000) extinction curve with $R_{V}=4$.1, because $A_{\mathrm{H} \alpha \text {,Calzetti }} / A_{\mathrm{H} \alpha \text {,Cardelli }}=1.03$ (Catalán-Torrecilla et al. 2015). The resulting pixel-by-pixel mean value of $A_{\mathrm{H} \alpha}$ is about 1 magnitude. Then, we apply this $A_{\mathrm{H} \alpha}$ to $\mathrm{H} \alpha$ maps to get the dust-corrected (or extinction-free) $\mathrm{H} \alpha$ maps. An example of this Balmer decrement method is shown in Figure 2.

We convert the dust-corrected $\mathrm{H} \alpha$ maps to the SFR surface density maps following the prescriptions in Calzetti et al. (2007), based on a stellar population model with $100 \mathrm{Myr}$ of constant SFR, solar metallicity, and an IMF that has a slope of -1.3 within $0.1<M_{*} / M_{\odot}<0.5$ and a slope of -2.3 within $0.5<M_{*} / M_{\odot}<120$ stellar mass range. The IMF for this SFR prescription is similar to a Kroupa (2001) IMF, which is a factor of 1.59 smaller than those derived from a Salpeter (1955) IMF within a mass range of $0.1-100 M_{\odot}$ (Madau \& Dickinson 2014). An example of the $\Sigma_{\text {SFR }}$ maps is shown as the second column of Figure 1.

As a check, we compare the SFR of extinction-corrected $\mathrm{H} \alpha$ emission that we derived above with the SFR derived from the ultraviolet (UV) emission plus total-infrared (TIR) emission from Catalán-Torrecilla et al. (2015). The UV emission traces the unobscured SFR, while the TIR emission compensates for the obscured SFR that is reradiated by dust. We do galaxy-bygalaxy comparisons by integrating our resolved SFR because the infrared data are unresolved. Since the $\mathrm{H} \alpha$ emission is more extended than the FoV of the CALIFA survey, we apply an aperture correction of 1.4 as suggested by Catalán-Torrecilla et al. (2015). In Figure 3, we show that both measurements are in agreement within a factor of $\sim 2$. 

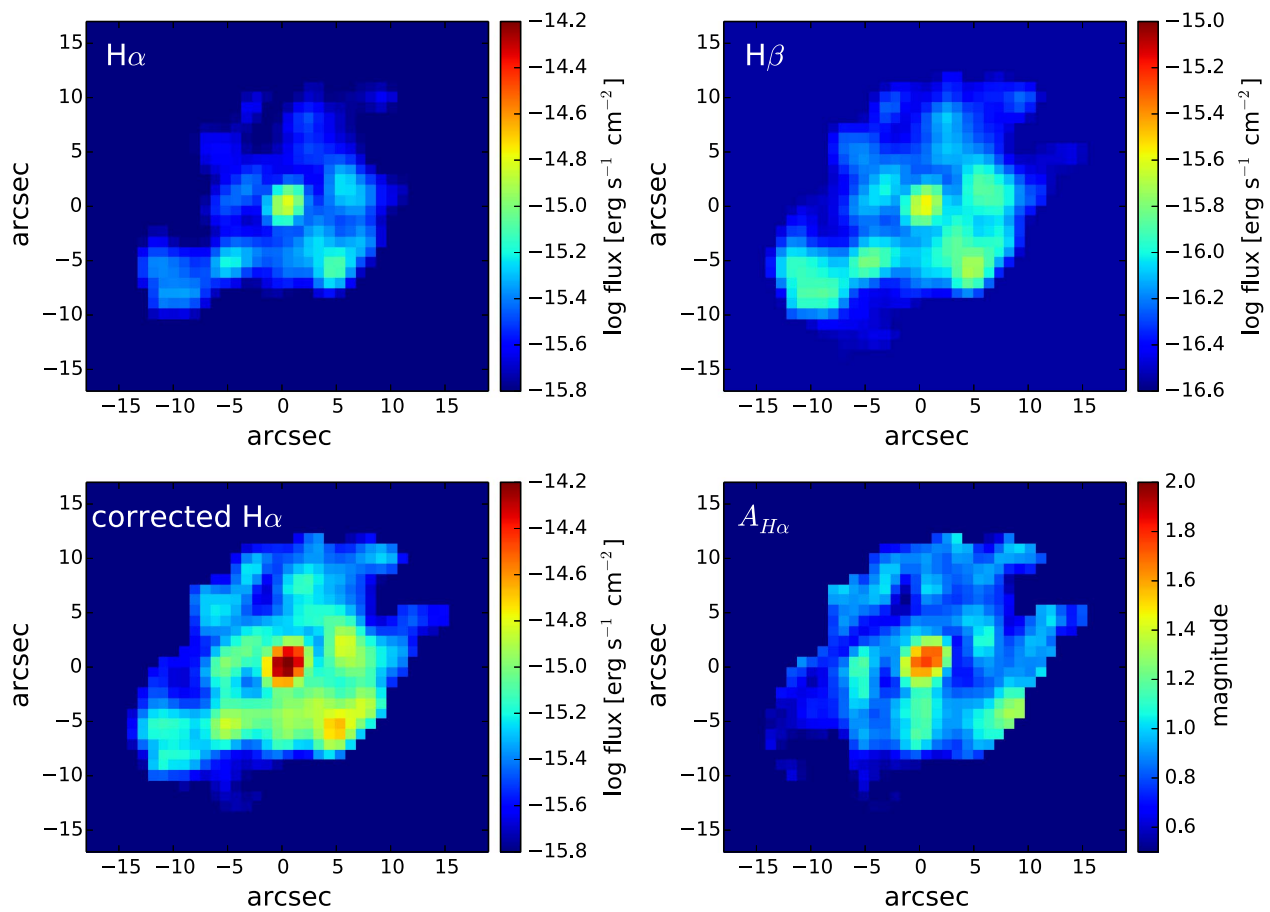

Figure 2. Application of the Balmer decrement method to NGC 2253. Top left: $\mathrm{H} \alpha$ fluxes. Top right: $\mathrm{H} \beta$ fluxes. Bottom right: dust extinction at $\mathrm{H} \alpha\left(A_{\mathrm{H} \alpha}\right)$. Bottom left: by correcting $A_{\mathrm{H} \alpha}$ to $\mathrm{H} \alpha$ fluxes reveals the dust-corrected $\mathrm{H} \alpha$ fluxes.

\subsubsection{The Gas-phase Metallicities}

We determine the gas-phase metallicities by using the emission line ratio of $\mathrm{O} \mathrm{III}[5007 \AA] / \mathrm{H} \beta$ and $\mathrm{N} \mathrm{II}[6583 \AA] / \mathrm{H} \alpha$ (i.e., the O3N2 method; Alloin et al. 1979; Pettini \& Pagel 2004). We use the following prescription from Marino et al. (2013).

$$
12+\log (\mathrm{O} / \mathrm{H})=8.533-0.214 \log \left(\frac{\mathrm{O} \text { III }}{\mathrm{H} \beta} \frac{\mathrm{H} \alpha}{\mathrm{N}_{\text {II }}}\right) .
$$

The coefficient of this method has been calibrated by using the electron temperature based measurements in $603 \mathrm{H}$ II regions extracted from literature and 3423 additional $\mathrm{H}$ II complexes from the CALIFA survey. The resolved metallicities in our sample range from 8.3 to 8.6, slightly below the Solar metallicity of 8.7 (Allende Prieto et al. 2001).

\subsubsection{The Stellar Ages and Mass Surface Densities}

We take the luminosity-weighted, stellar population ages and the dust-corrected, stellar mass surface densities $\left(\Sigma_{*}\right)$ from the data products of Pipe3D version 2.2 (Sánchez et al. 2016). Briefly, the data products are derived from the best fit of stellar spectra from a combination of the GRANADA (Martins et al. 2005) and MILES libraries (Sánchez-Blázquez et al. 2006; Vazdekis et al. 2010; Falcón-Barroso et al. 2011), which cover 39 grids of stellar ages (from $1 \mathrm{Myr}$ to $13 \mathrm{Gyr}$ ) and 4 grids of stellar metallicities $\left(Z / Z_{\odot}=0.2,0.4,1\right.$ and 1.5$)$. We convert the $\Sigma_{*}$ maps from a Salpeter (1955) IMF to a Kroupa (2001) IMF by dividing it by a factor of 1.59 (Madau \& Dickinson 2014).

\section{Sample Selection}

We select 52 galaxies from 126 EDGE galaxies based on the following three criteria. (1) They are not dominated by AGN

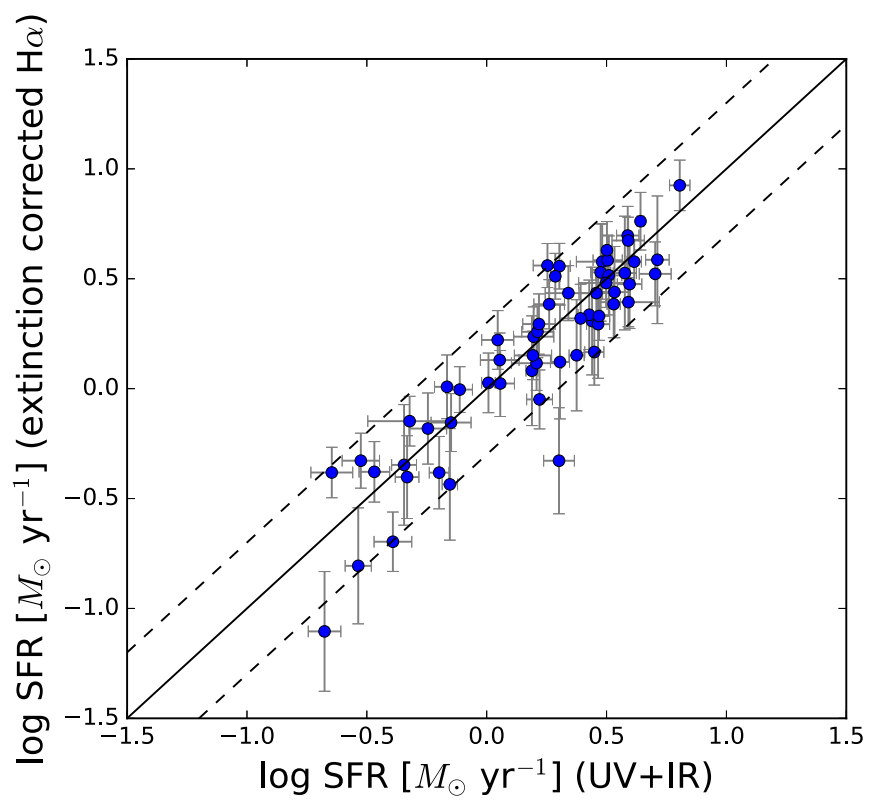

Figure 3. Comparison of SFR measurements from extinction-corrected $\mathrm{Ho}$ (this paper) and UV+IR from Catalán-Torrecilla et al. (2015). We apply aperture correction for our SFR measurement as suggested by CatalánTorrecilla et al. (2015). The solid line is the one-to-one relationship, while the dashed line is 0.3 dex away from the solid line. The uncertainties of SFR measurement in this paper is calculated using the error propagation from the uncertainties in $\mathrm{H} \alpha$ and $\mathrm{H} \beta$ measurements. A $20 \%$ uncertainty due to SFR calibration (Calzetti et al. 2007) has been included as well.

and LINER. (2) They have sufficient SFR and CO detection that cover both the centers and the disk. (3) The inclination (i) is less than $75^{\circ}$. The inclinations are taken from the following sources, ordered by priority: (1) the best fit of $\mathrm{CO}$ rotation curve, whenever it is possible (R. C. L. Levy et al. 2017, in preparation), (2) from the shape of the outer isophote, or (3) 
from the HyperLEDA catalog (Makarov et al. 2014). A list of the galaxy sample is tabulated in Appendix A.

We exclude AGN and LINER emission regions based on $\mathrm{N}$ II $/ \mathrm{H} \alpha$ and $\mathrm{O}$ III $/ \mathrm{H} \beta$ line ratios (i.e., the BPT diagram; Baldwin et al. 1981; Kewley \& Dopita 2002; Kauffmann et al. 2003). Any data points above the demarcation line of Kewley \& Dopita (2002) are blanked. We also blank any regions that have $\mathrm{H} \alpha$ equivalent width less than $6 \AA$, because $\sim 80 \%$ of stars in those regions are older than $\sim 500 \mathrm{Myr}$, and hence, not associated to star-forming regions (Sánchez et al. 2014). Note that the LINER emission region is not only concentrated in the center, but also in the disk, possibly due to photoionization from AGB stars (Singh et al. 2013; Belfiore et al. 2016). A galaxy is removed from the samples if all pixels in the center (i.e., within $0.1 R_{25}$ ) are AGN/LINER-like emission. Based on that criterion, 31 galaxies from the EDGE sample are removed.

We further remove 17 galaxies that do not have sufficient $\mathrm{CO}$ or SFR detection in the centers or in the disks, because measurement of $\tau_{\text {dep }}$ is severely contaminated by nondetection. If a galaxy has less than two detected pixels in the center or in the disk, then that galaxy is removed from the sample. Lastly, 26 galaxies with $i \gtrsim 75^{\circ}$ (equivalents to the ratio of minor to major axes of less than 0.25 ) are removed because highly inclined galaxies yield few sampling points along the minor axis, resulting in a deprojected beam elongated parallel to the minor axis in the plane of the galaxy, and high uncertainty in the estimation of dust extinction.

Our final sample has stellar masses $\left(M_{*}\right)$ from $4 \times 10^{9}$ to $2 \times 10^{11} M_{\odot}$, molecular gas masses $\left(M_{\mathrm{mol}}\right)$ from $8 \times 10^{7}$ to $1 \times 10^{10} M_{\odot}$, and gas-phase metallicities $(12+\log [\mathrm{O} / \mathrm{H}])$ from 8.4 to 8.6 dex. Our sample consists of 50 spirals (Hubble type from $\mathrm{Sa}$ to $\mathrm{Sd}$ ) and 2 early-types, 24 of which are barred and 7 of them are interacting (Barrera-Ballesteros et al. 2015). The ranges in the stellar and molecular gas masses are comparable to the unresolved survey of COLDGASS (Saintonge et al. 2011a, 2011b). In addition, we have a comparable number of galaxies and cover farther distance in the local volume $(26 \lesssim d \lesssim 169 \mathrm{Mpc})$ than previous resolved surveys, such as BIMA SONG (44 galaxies; $2 \lesssim d \lesssim 26 \mathrm{Mpc}$; Helfer et al. 2003), Nobeyama CO Survey (40 galaxies; $d<25$ Mpc; Kuno et al. 2007), CARMA STING (14 galaxies; $5 \lesssim d \lesssim 43 \mathrm{Mpc}$; Rahman et al. 2012), JCMT NGLS (155 galaxies; $d<25$ Mpc; Wilson et al. 2012), and HERACLES (48 galaxies; $3 \lesssim d \lesssim$ 15 Mpc; Schruba et al. 2012; Leroy et al. 2013). Thus, our sample bridges the gap between nearby and higher redshift galaxies.

\section{Results}

In Figure 4, we show the KS relation for molecular gas. The data points are from pixel measurements (detected both in SFR and CO) in 52 galaxies. The median values of $\Sigma_{\text {SFR }}$ for a given bin of $\Sigma_{\text {mol }}$ are marked as black dots, while the constant values of $\tau_{\text {dep }}=1,2$, and $4 \mathrm{Gyr}$ are indicated. There is a tendency that the high $\Sigma_{\text {mol }}$ region (top right in Figure 4) has a slightly shorter $\tau_{\text {dep }}$ than the low $\Sigma_{\text {mol }}$ region (i.e., the best-fit slope is slightly larger than unity). Since galactic centers have higher $\Sigma_{\text {mol }}$ than that in the disks, this indicates that the centers have shorter $\tau_{\text {dep }}$ than in the disks.

In order to study the variation of $\tau_{\text {dep }}$ between the galactic centers and disks, we need to separate the central region of a galaxy. To do so, we define the center as a region within

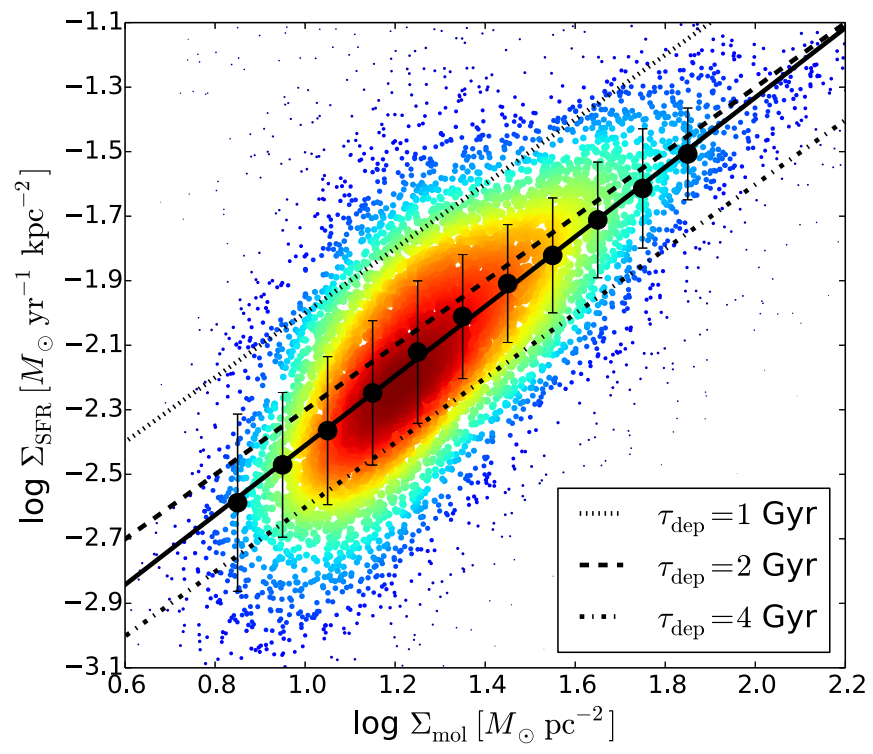

Figure 4. Relationship between $\Sigma_{\mathrm{mol}}$ and $\Sigma_{\mathrm{SFR}}$ for 52 galaxies in our sample. The data points are pixel-by-pixel measurements $\left(2^{\prime \prime} \times 2^{\prime \prime}\right)$, with colors and point sizes coded by the density of data points. The black dots are the median values of $\Sigma_{\mathrm{SFR}}$ within bins of $\Sigma_{\mathrm{mol}}$. A linear fit to the black dots is given by the solid black line. This linear fit has a slope of $1.08 \pm 0.01$ and an intercept point of $-3.49 \pm 0.02$. The dotted, dashed, and dash-dotted lines correspond to $\tau_{\text {dep }}=1,2$, and 4 Gyr, respectively.

$0.1 R_{25}$ from the galactic nucleus, and the disk as a region between $0.1 R_{25}$ and $0.7 R_{25}$. Therefore, $\tau_{\text {center }}$ and $\tau_{\text {disk }}$ are the median of $\tau_{\text {dep }}$ over all detected pixels in the center and in the disk, respectively. If the median or the whole value of $\tau_{\text {dep }}$ in a galaxy is used, it means we cover both the center and the disk, and we refer to it as $\tau_{\text {dep,med }}$. If the number of detected pixels in the disks is much larger than those in the centers, then the values of $\tau_{\text {dep,med }}$ is similar to $\tau_{\text {disk }}$. We adopt $0.7 R_{25}$ as the outermost radius because $\mathrm{CO}$ is hardly detected beyond that radius.

The radial distance to the galactic nucleus is calculated using the assumption that the molecular gas lies on the galactic midplane, without warp, isophotal twist, and misalignment. Since each galaxy has a different physical size in kiloparsecs, sometimes we normalize the radius with respect to $R_{25}$, i.e., the radius where the surface brightness is $25 \mathrm{mag} \operatorname{arcsec}^{-2}$ in the $B$-band. We adopt the values of $R_{25}$ from the HyperLEDA catalog. The scaling relation between $R_{25}$ and the stellar scale length $\left(l_{*}\right)$ is $R_{25}=(4.6 \pm 0.8) l_{*}$ (Leroy et al. 2008). Unless otherwise stated, throughout this paper, we focus on the starforming regions detected in both $\mathrm{CO}\left(\Sigma_{\mathrm{mol}} \gtrsim 10 M_{\odot} \mathrm{pc}^{-2}\right)$ and $\mathrm{H} \alpha$ on a pixel-by-pixel basis (an $\sim$ kiloparsec scale).

\subsection{Depletion Time in the Centers and in the Disks}

Since CO emission is patchy, not all regions within a galaxy are detected in $\mathrm{CO}$ and $\mathrm{H} \alpha$. To accrue more signal-to-noise and to get a better radial coverage across the sample, we aggregate the $\tau_{\text {dep }}$ measurements as a function of $r / R_{25}$ for all galaxies. By doing this measurement for the $\mathrm{CO}$ detections only, we focus on regions that, like most galaxy centers, are dominated by molecular gas $\left(\Sigma_{\mathrm{mol}} \geqslant 10 M_{\odot} \mathrm{pc}^{-2}\right)$, and where similar starformation mechanisms are likely to operate. In Figure 5, $\tau_{\text {dep }}$ in each detected pixel are plotted as a function of radius. The median value of $\tau_{\text {dep }}$ is $2.4 \mathrm{Gyr}$ with $\sim 0.5$ dex scatter. This value is in line with the previous measurements in nearby 


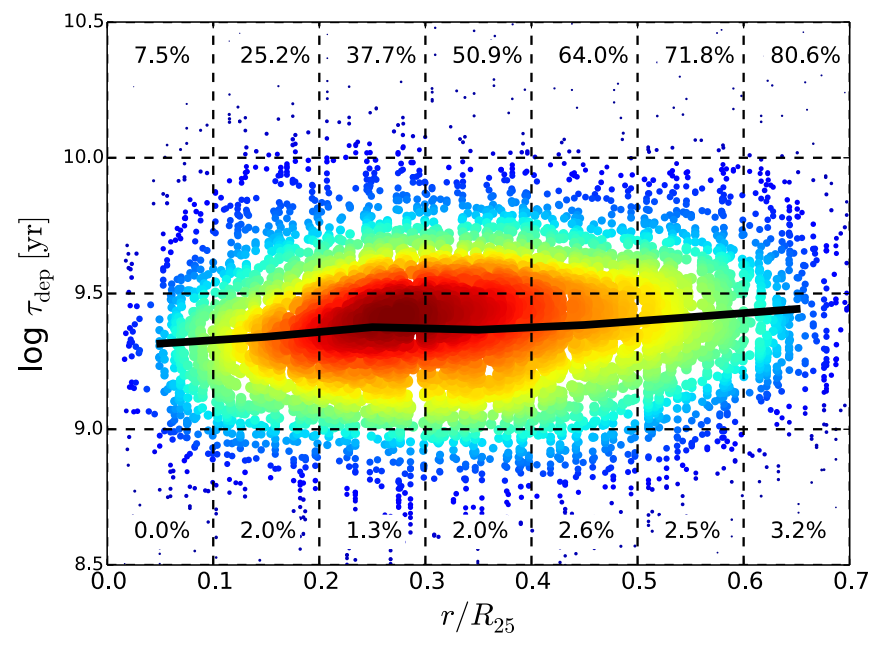

Figure 5. Depletion time as a function of radius, aggregated over all detected regions in the sample. The data points are the pixel-by-pixel $\left(2^{\prime \prime} \times 2^{\prime \prime}\right)$ measurements. The colors and sizes of points represent the global density of the data points and the solid line is the median value of $\tau_{\text {dep }}$ in radial bins. On the top and bottom of the figure, we label the fractions of nondetection pixels that correspond to upper and lower limits in $\tau_{\text {dep }}$, respectively. Upper limits in $\tau_{\text {dep }}$ are pixels with known SFRs but CO is not detected, and vice versa for lower limits. The $\mathrm{H} \alpha$ measurements are more sensitive than the $\mathrm{CO}$ maps, therefore, the fractions of upper limits are higher than the fractions of lower limits at any radius.

galaxies (e.g., Bigiel et al. 2011; Rahman et al. 2012; Leroy et al. 2013). Pointings in the center, however, have shorter $\tau_{\text {dep }}$ than those in the disk. However, the dip of $\tau_{\text {center }}$ does not occur in all galaxies in the sample, and becomes more prominent when we separate those galaxies from the rest of the sample (see Section 4.2).

In Figure $6, \tau_{\text {center }}$ and $\tau_{\text {disk }}$ for each galaxy are shown. The ratio between $\tau_{\text {center }}$ and $\tau_{\text {disk }}$ in our sample can reach a factor of $\sim 10$, but the ratio in most galaxies is between unity and a factor of 3 . The scatter in $\log \left(\tau_{\text {center }} / \tau_{\text {disk }}\right)$ is larger in the high stellar and molecular gas masses regime. We investigate whether the variation of $\tau_{\text {center }}$ relative to $\tau_{\text {disk }}$ is correlated to the global properties of galaxies, namely the stellar masses $\left(M_{*}\right)$, the molecular gas masses $\left(M_{\mathrm{mol}}\right)$, the Hubble types, the gas-phase metallicities, and the age of stellar populations. We adopt RC3 de Vaucouleurs et al. (1991) indices from the HyperLEDA catalog as morphological types. For the oxygen abundance and the age of the stellar population, we use their median value within a $1 \pm 0.2$ effective radius $\left(R_{e}\right)$ because Sánchez et al. (2016) suggest that the value at $R_{e}$ is a good representation for a galaxy.

We do not find a correlation between $\log \left(\tau_{\text {center }} / \tau_{\text {disk }}\right)$ and morphology, gas-phase metallicity, or age of stellar populations at $R_{e}$, probably because we have a limited range in morphology (96\% of our samples are spirals) and gas-phase metallicity (only $\sim 0.2$ dex of variations). Furthermore, the age of stellar populations at $R_{e}$ reflect the value in the disks, where $\tau_{\text {disk }}$ does not vary as much as $\tau_{\text {center. }}$. If we measure the stellar age in the center, however, galaxies with low values of $\log \left(\tau_{\text {center }} / \tau_{\text {disk }}\right)$ have younger ages for stellar populations (see Section 5.4). There is also no significant correlation between $\tau_{\text {center }} / \tau_{\text {disk }}$ and $M_{*}, M_{\mathrm{mol}}$, and $M_{\mathrm{mol}} / M_{*}$ (Figure 6), as indicated by low values of Kendall (1938) $\tau$-coefficient.

It should be noted that three galaxies with the lowest values of $\log \left(\tau_{\text {center }} / \tau_{\text {disk }}\right)$ are interacting galaxies (marked as black squares in Figure 6). In addition, barred galaxies, marked as black diamonds in Figure 6 (identified from the photometric fit of Méndez-Abreu et al. 2017, or from the HyperLEDA catalog), tend to have lower values of $\log \left(\tau_{\text {center }} / \tau_{\text {disk }}\right)$ than unbarred galaxies. The mean values of $\log \left(\tau_{\text {center }} / \tau_{\text {disk }}\right)$ for interacting and barred galaxies are $-0.42 \pm 0.51$ and $-0.22 \pm 0.28$, while the corresponding value for unbarred galaxies is $-0.03 \pm 0.35$. This indicates that perturbed systems may enhance the star-formation efficiency in the center.

\subsection{Separations of Galaxies into Three Groups of $\tau_{d e p}$}

To see a clear variation of $\tau_{\text {center }}$ with respect to $\tau_{\text {disk }}$, we separate galaxies into three groups based on their log ( $\left.\tau_{\text {center }} / \tau_{\text {disk }}\right)$ values. The three groups of $\tau_{\text {dep }}$ are the following. (1) Galaxies with falling $\tau_{\text {center }}$, defined as those with $\log$ $\left(\tau_{\text {center }} / \tau_{\text {disk }}\right)<-0.26$ dex, represent $26.9 \%$ of the galaxy sample. (2) Galaxies with rising $\tau_{\text {center }}$, defined as those with $\log \left(\tau_{\text {center }} / \tau_{\text {disk }}\right)>0.26$ dex, represent $11.5 \%$ of the galaxy sample. (3) The rest of them (61.6\% of the sample) have log $\left(\tau_{\text {center }} / \tau_{\text {disk }}\right)$ within \pm 0.26 dex, which we defined as flat $\tau_{\text {dep }}$. We list the values of $\tau_{\text {dep }}$ in the centers, disks, and whole galaxy (median) in Appendix A, where we use the notation "drop," "rise," and "flat" for these three groups. In this respect, we expand the previous finding that galactic centers have shorter $\tau_{\text {dep }}$ than that in the disks (Leroy et al. 2013) to include galactic centers that have similar, and even, longer $\tau_{\text {center }}$ compared to $\tau_{\text {disk }}$. The results of this segregation are shown in the top row of Figure 7.

We use 0.26 dex as a separator between three different groups of $\tau_{\text {dep }}$ because this value is the standard deviation of resolved $\tau_{\text {dep }}$ measurements within $0.7 R_{25}$. This value also coincides with what was found in several galaxies of the HERACLES sample, which show a dip of $\tau_{\text {center }}$ by about 0.2 dex relative to $\tau_{\text {disk }}$ (for a constant CO-to- $\mathrm{H}_{2}$ conversion factor; Leroy et al. 2013). However, keep in mind that the variation of $\tau_{\text {center }}$ is continuous, i.e., there is no clear separation or clustering between those three groups (see Figure 6). This classification of galaxies into three groups is just an approach to see a difference between $\tau_{\text {center }}$ and $\tau_{\text {disk }}$ in some galaxies.

We check how robust this classification is after the inclusion of upper and lower limits of $\tau_{\text {dep }}$ in Appendix B. The number of galaxies in the drop $\tau_{\text {center }}$ group reduces from 14 to 12 after the inclusion of non-detections as $1 \sigma_{\mathrm{rms}}$ and increases from 14 to 20 after the inclusion of nondetections as $2 \sigma_{\text {rms. }}$. We refer to those numbers as the uncertainties of our classification, i.e., the number of galaxies in the drop $\tau_{\text {center }}$ group is $14_{-2}^{+6}$. For the flat and rising $\tau_{\text {center }}$ groups, the corresponding numbers are $32_{-4}^{+2}$ and $6_{-2}^{+0}$, respectively. About $88.5 \%$ of the sample does not change group after the inclusion of nondetections as $1 \sigma_{\text {rms. }}$. This means the numbers of galaxies in each group are quite robust.

In Appendix $\mathrm{C}$, we check whether the drop of $\tau_{\text {center }}$ is affected by varying physical resolutions from 1 to $3 \mathrm{kpc}$. This is equivalent to placing galaxies at a farther distance. We found that the drop of $\tau_{\text {center }}$ is more prominent in a scale of $1 \mathrm{kpc}$. This means the number of galaxies in the drop $\tau_{\text {center }}$ group is likely to be larger if we have a resolution better than $1 \mathrm{kpc}$.

In the bottom row of Figure 7, we show each of the three groups in the absolute scale of $\tau_{\text {dep }}$ (in years). It shows that the galactic centers in the drop (rise) $\tau_{\text {center }}$ groups form stars more (less) efficiently than those in the flat $\tau_{\text {dep }}$ group, i.e., their locations in the KS diagram lie above (below) the disks. The values of $\tau_{\text {center }}$ in the drop $\tau_{\text {dep }}$ group $(\approx 1 \mathrm{Gyr})$ are not only lower relative to $\tau_{\text {disk }}$, but also in the absolute sense. Therefore, 

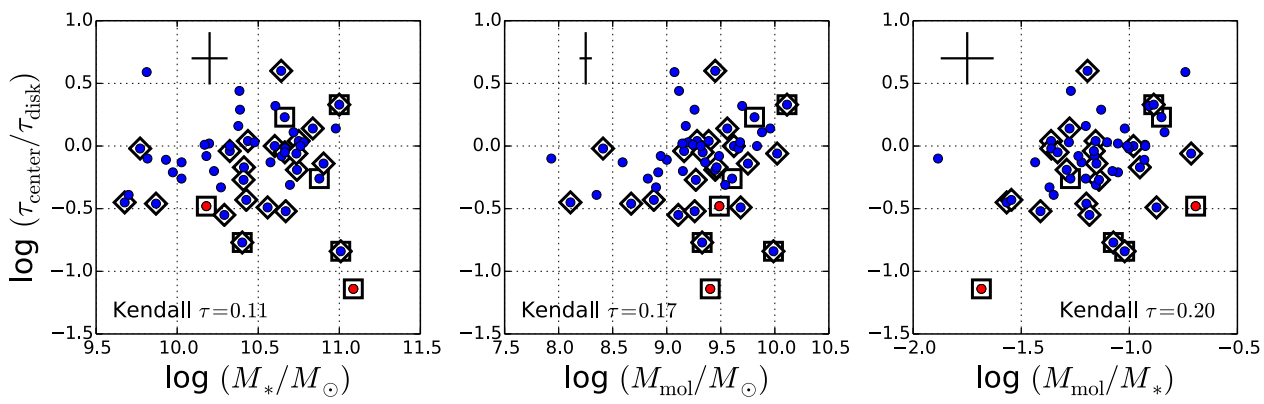

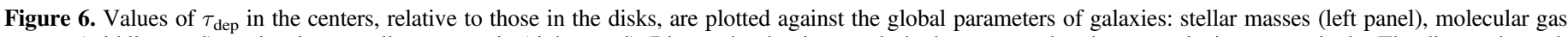

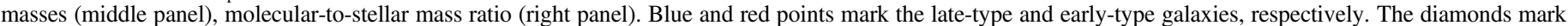

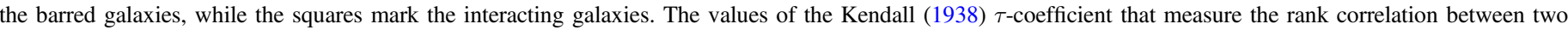

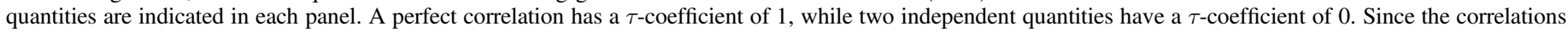
are not significant, we do not plot the best-fit line. The crosses represent the typical error bars of the data points.

those galactic centers resemble an intermediate regime between the disks and starbursts.

\subsection{The Local Properties}

Is the variation of $\tau_{\text {dep }}$ between the centers and the disks driven by SFR, molecular gas, or both? In Figure 8, we show that there is an anti-correlation between $\log \left(\tau_{\text {center }} / \tau_{\text {disk }}\right)$ and $\log$ $\left(\sum_{\mathrm{SFR}}^{\text {center }} / \Sigma_{\mathrm{SFR}}^{\text {disk }}\right)$, but no correlation between $\log \left(\tau_{\text {center }} / \tau_{\text {disk }}\right)$ and $\log \left(\sum_{\text {mol }}^{\text {center }} / \Sigma_{\text {mol }}^{\text {disk }}\right)$. This means the drop of $\tau_{\text {center }}$ is due to higher $\Sigma_{\text {SFR }}$, not lower $\Sigma_{\text {mol }}$ in the center. In other words, the centers can have any values of $\Sigma_{\text {mol }}$, but those with higher $\Sigma_{\text {SFR }}$ are associated with the drops of $\tau_{\text {center. }}$. However, we should be cautious that the range of $\Sigma_{\text {mol }}$ variations $(\sim 1$ dex $)$ is smaller than the range of $\Sigma_{\mathrm{SFR}}$ variations $(\sim 2 \mathrm{dex})$.

Then, why do some centers have higher $\Sigma_{\mathrm{SFR}}$, irrespective of the $\Sigma_{\text {mol }}$ value? In thermal and dynamical equilibrium, the weight of the ISM in the vertical gravitational field of stars and gas is balanced by the pressure created by momentum and energy from stellar feedback (Ostriker et al. 2010; Kim et al. 2011, 2013; Ostriker \& Shetty 2011). Therefore, we expect a relation between $\Sigma_{\mathrm{SFR}}$ (which sets the thermal, turbulent, and magnetic pressure via feedback) and $\Sigma_{*}$ (which sets the ISM weight). Interestingly, in the right panels of Figure 8 , we see that $\log \left(\tau_{\text {center }} / \tau_{\text {disk }}\right)$ correlates with the ratio of the mean values of $\Sigma_{*}$ between the center and the disk. Galaxies with higher ratios of central $\Sigma_{*}$ relative to those in the disks, have a drop of $\tau_{\text {center. }}$. Since $\Sigma_{*}$ is one of the determining factors for hydrostatic pressure (Blitz \& Rosolowsky 2004, 2006), this means the drops of $\tau_{\text {center }}$ are associated with high ISM pressure. Indeed, previous observations showed that the galactic center is a high pressure region (Spergel \& Blitz 1992; Oka et al. 2001; Rosolowsky \& Blitz 2005). This result suggests that the star-formation efficiency depends on the local environment within a galaxy.

\section{Discussion}

\subsection{The CO-to- $\mathrm{H}_{2}$ Conversion Factor}

How is the variation of $\tau_{\text {center }}$ affected by the change in the CO-to- $\mathrm{H}_{2}$ conversion factor $\left(\alpha_{\mathrm{CO}}\right)$ ? In general, there are two scenarios where $\alpha_{\mathrm{CO}}$ varies (Bolatto et al. 2013). First, the dependence of $\alpha_{\mathrm{CO}}$ with gas metallicity-a lower gas metallicity needs a higher $\mathrm{H}_{2}$ column density to shield the gas until it reaches sufficient extinction for $\mathrm{CO}$ to exist (e.g., Leroy et al. 2007, 2011). However, the variation of metallicity from center to disk within a galaxy is very small $(\sim 0.1$ dex;
Figure 9), so that metallicity is unlikely to induce a significant variation on $\alpha_{\mathrm{CO}}$. Furthermore, in the group that shows a drop of $\tau_{\text {center }}$, metallicities slightly rise toward the center, which means $\alpha_{\mathrm{CO}}$ is slightly lower in the center than in the disk. If we take this effect into account, it would only exaggerate the drop of $\tau_{\text {center. }}$.

The second source of $\alpha_{\mathrm{CO}}$ variations is the $\mathrm{CO}$ emission from diffuse gas that is bound by the gravitational potential of stars and gas. Hence, the velocity dispersion of this diffuse gas $\left(\sigma_{\mathrm{CO}, \text { diff }}\right)$ reflects the additional stellar gravitational potential (Bolatto et al. 2013). This effect increases the CO luminosity $\left(L_{\mathrm{CO}}\right)$ per unit molecular gas mass because $L_{\mathrm{CO}}$ is proportional to the brightness temperature $\left(T_{B}\right)$ and $\sigma_{\mathrm{CO} \text {,diff }}$ (assuming $\mathrm{CO}$ is optically thick throughout the medium). Bolatto et al. (2013) and Sandstrom et al. (2013) suggest that the variation of $\alpha_{\mathrm{CO}}$ is related to the total surface density due to stars and gas as $\alpha_{\mathrm{CO}}$ $\propto \Sigma_{\text {total }}^{-\gamma}$, where $\gamma \approx 0.5$ for $\Sigma_{\text {total }}>100 M_{\odot} \mathrm{pc}^{-2}$. Applying this prescription for $\alpha_{\mathrm{CO}}$ would exaggerate the drop of $\tau_{\text {center }}$ and result in more galaxies in the group of $\tau_{\text {center }}$ drops.

\subsection{Metallicity Gradients}

It is interesting that the metallicity in the drop $\tau_{\text {center }}$ group is rising toward the centers, while the metallicity profiles in the other two groups are flattening toward the centers (Figure 9). In the CALIFA sample, Sánchez-Menguiano et al. (2016) found the variation of metallicity gradients for different stellar masses: the metallicity gradient in higher mass galaxies is flattening in the center, while the metallicity gradient in lower mass galaxies is rising toward the center. Since the drop of $\tau_{\text {dep }}$ is more prominent in the lowest mass bin (Figure 10), the variation of metallicity gradients in Figure 9 is possibly driven by their correlation with stellar masses. However, it remains unknown why the metallicity gradient depends on the stellar masses.

An alternative interpretation of steeper metallicity gradient is an enhancement of SFR per unit gas mass in the center (i.e., a low value of $\tau_{\text {center }}$ ) leads to a more metal enrichment than in the disk. Unlike stellar metallicity, gas-phase metallicity is more sensitive to the recent star-formation activities, and hence, reflects the current value of $\tau_{\text {center}}$. However, the center is not a closed-box system because of inflowing gas from the disk and outflowing gas driven by the stellar feedback. Furthermore, the gas-phase metallicity is also determined by the star-formation history, not only the current star formation. Therefore, the rising gradient of metallicity in the short $\tau_{\text {center }}$ group is not clearly understood. 

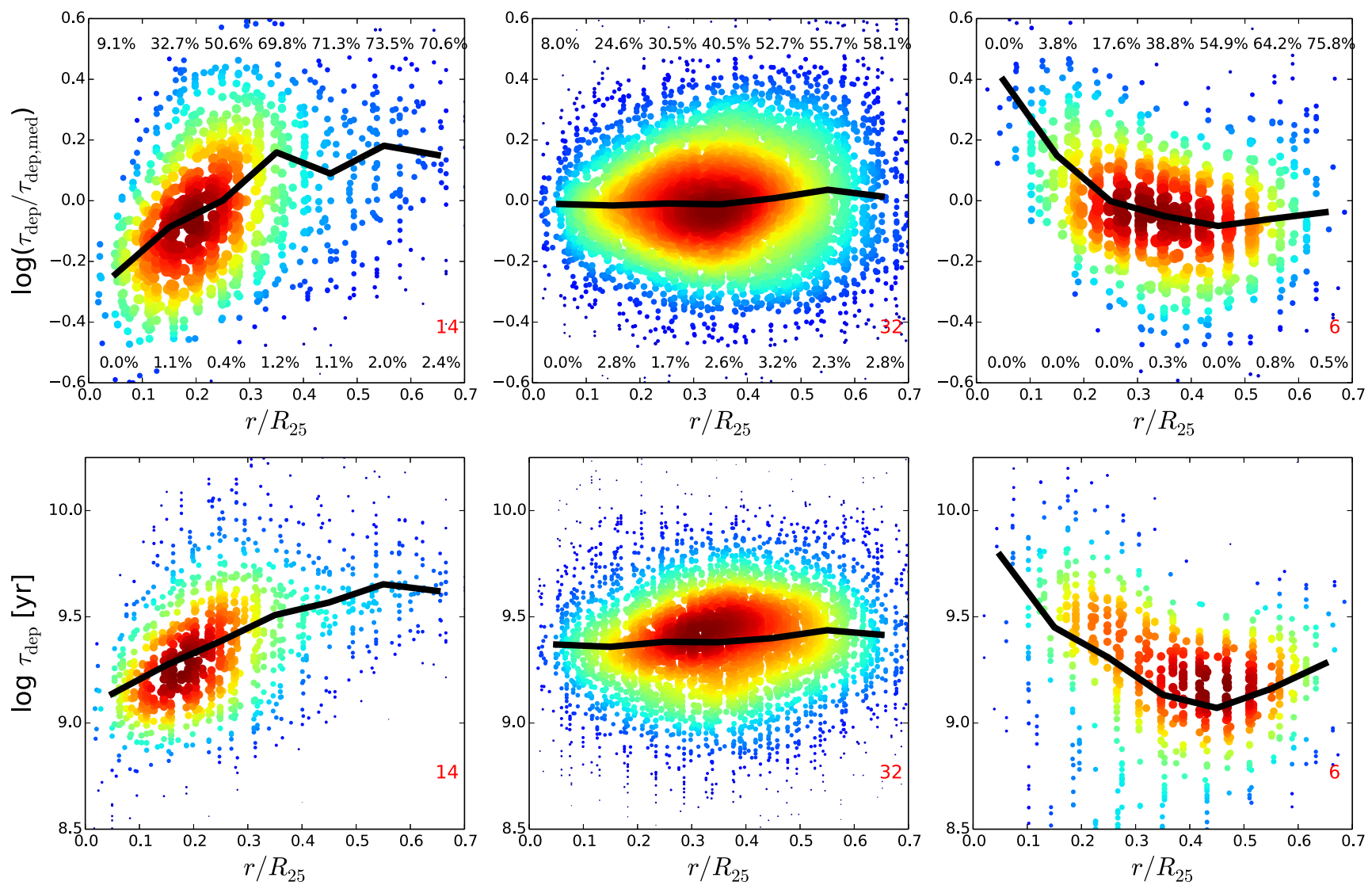

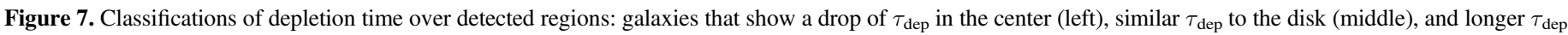

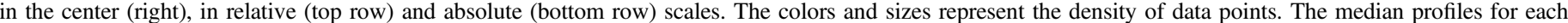

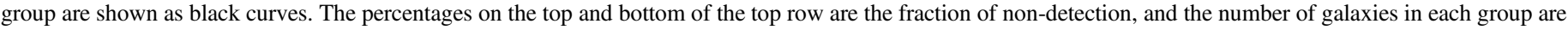

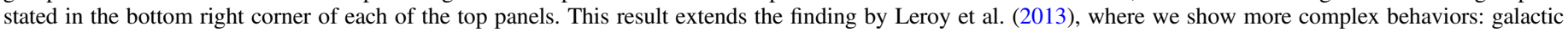
centers can have shorter, similar, or longer $\tau_{\text {dep }}$ with respect to the disk.
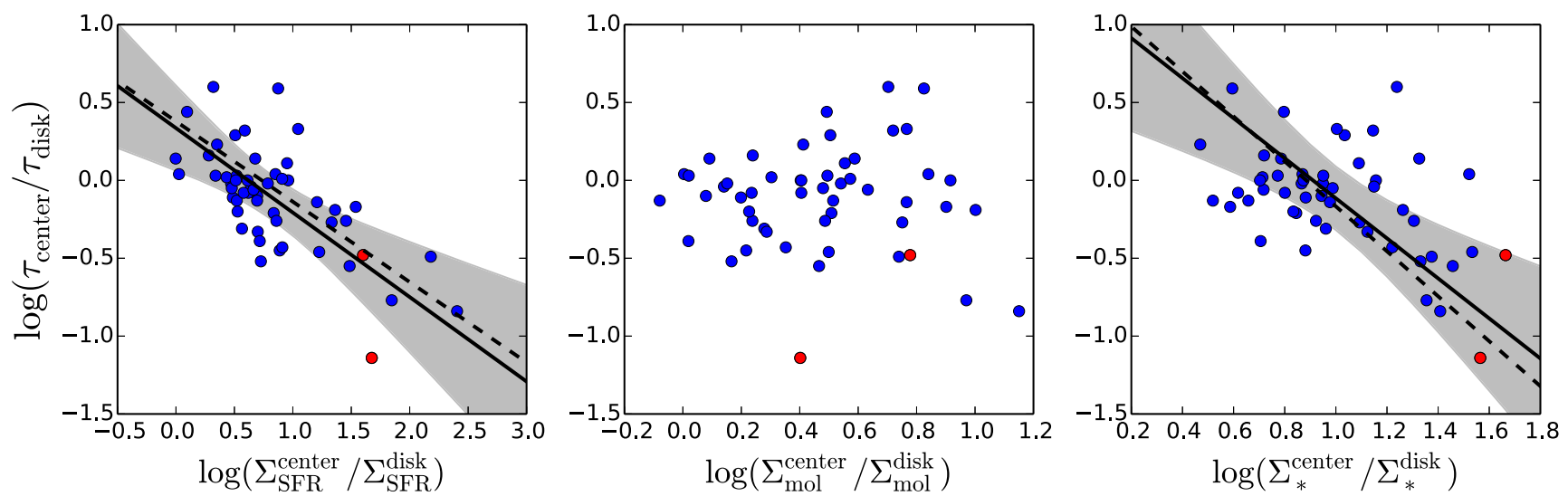

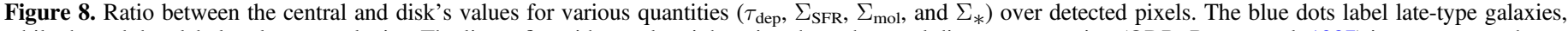

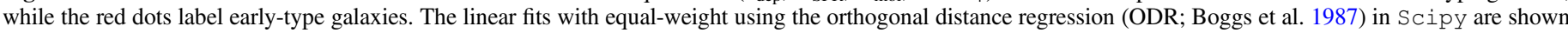

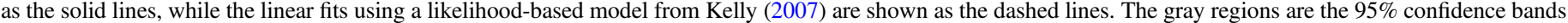

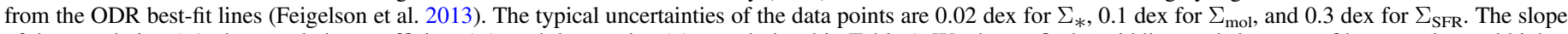

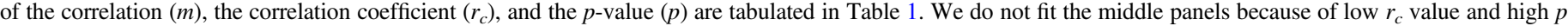
value, indicative of no correlation between $\log \left(\tau_{\text {center }} / \tau_{\text {disk }}\right)$ and $\log \left(\Sigma_{\text {mol }}^{\text {center }} / \Sigma_{\text {mol disk }}^{\text {dis }}\right)$.

\subsection{The Size of the Molecular Disk}

In Figure 7, we see that the distribution of data points in the short $\tau_{\text {center }}$ group is more concentrated toward the center, compared to those in the flat $\tau_{\text {dep }}$ group. This gives a clue that the size of the molecular disk in the short $\tau_{\text {center }}$ group may be smaller (more compact). In order to quantify the compactness of the molecular gas and stellar distributions, we calculate the half-mass-radius of molecular gas $\left(R_{1 / 2}^{\mathrm{mol}}\right)$ and stars $\left(R_{1 / 2}^{*}\right)$ from the cumulative distribution of $\Sigma_{\text {mol }}$ and $\Sigma_{*}$ as a function of radius (Bolatto et al. 2017).

In Figure 11, we plot $\log \left(\tau_{\text {center }} / \tau_{\text {disk }}\right)$ against $R_{1 / 2}^{\text {mol }}$ (left panel) and $R_{1 / 2}^{*}$ (right panel). It turns out that galaxies in the 

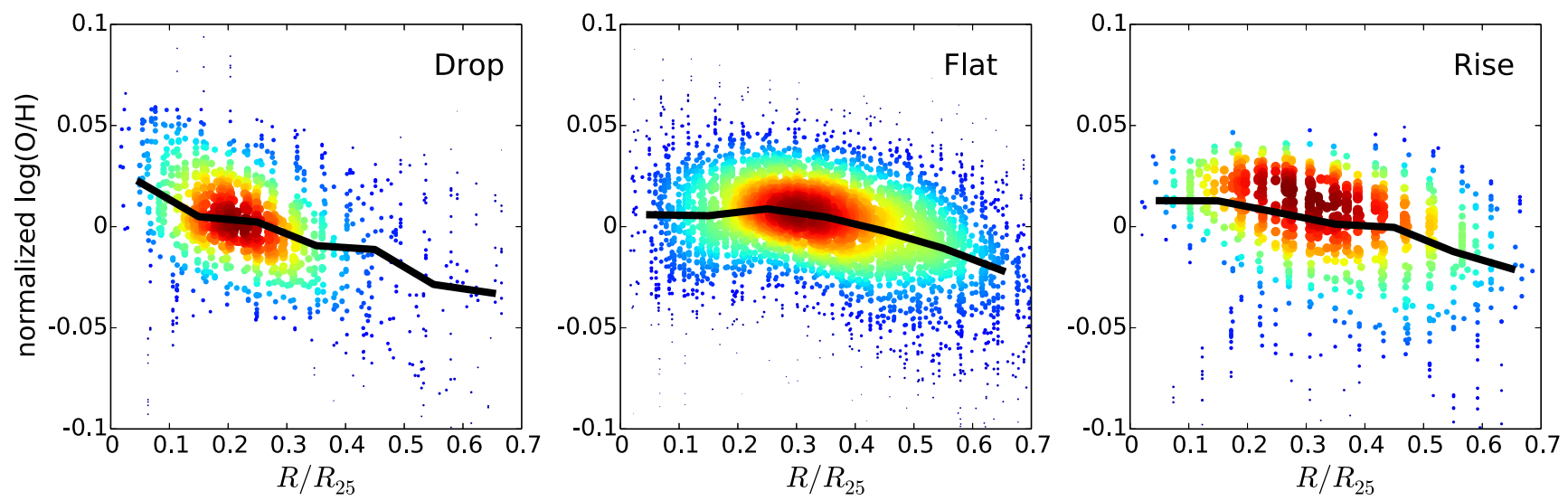

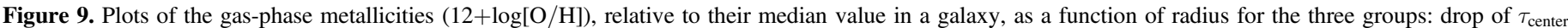

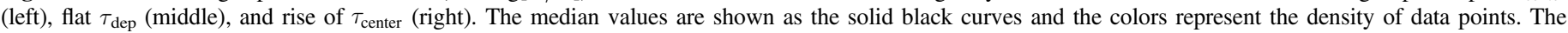
galaxies that show drops of $\tau_{\text {center }}$ have a steeper gradient of metallicity than the other two groups.
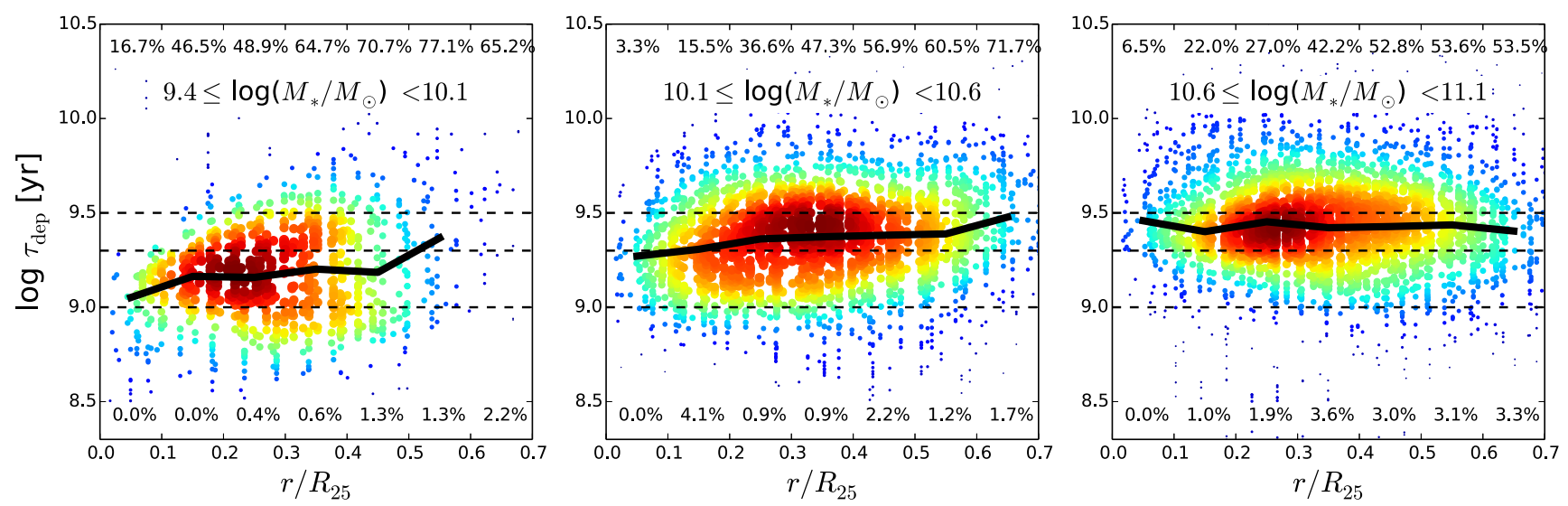

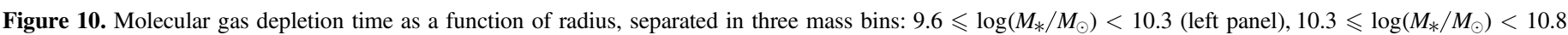

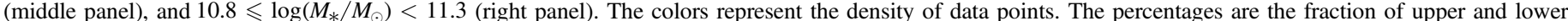

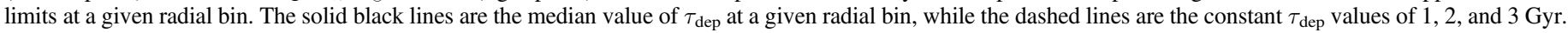
This figure shows that the drop of $\tau_{\text {dep }}$ in the centers is more prominent in the lowest mass bin.

drop $\tau_{\text {center }}$ group have smaller $R_{1 / 2}^{\mathrm{mol}}$ and $R_{1 / 2}^{*}$ than those in the other two groups (quantified in Table 2). About $75 \%$ of galaxies in the drop $\tau_{\text {center }}$ group are disturbed systems, compared to only $44 \%$ and $40 \%$ for the flat and rise $\tau_{\text {center }}$ groups, respectively. This gives a clue that the driver of physical size of the stellar and molecular gas distribution (maybe bars and interactions) is linked to the cause of $\tau_{\text {dep }}$ variation in the centers. We suspect that the bar drives the gas inward toward the center (or in the case of interacting galaxies, the gas loses its angular momentum). This radial gas influx increases the pressure, resulting in higher star-formation efficiency in the galactic center.

\subsection{A Burst of Star Formation}

For galaxies in the drop $\tau_{\text {center }}$ group, there may be central starburst activity on scales below our resolution as indicated by the stellar population ages. There are at least two tracers of the stellar population ages: the UV-to-H $\alpha$ ratio (e.g., Leroy et al. 2012; Weisz et al. 2012) and the age derived from the stellar population synthesis (which is available in the IFU data products of Sánchez et al. 2016). Since we do not have the resolved UV maps in hand, we rely on the second tracer. In Figure 12, we show the histogram of the luminosity-weighted ages of stellar populations in the centers $\left(r<0.1 R_{25}\right)$ for each $\tau_{\text {dep }}$ group. It turns out that the centers in the drop $\tau_{\text {center }}$ group (left panel) tend to have younger ages of stellar populations $(\approx 2.1 \pm 1.1 \mathrm{Gyr})$ than the other two groups $(\approx 2.5 \pm 1.6 \mathrm{Gyr}$ and $\approx 3.1 \pm 1.6 \mathrm{Gyr}$; middle and right panels).

We do a Kolmogorov-Smirnov test to check whether the age distributions in each group can be drawn from the same underlying distribution. The $p$-values between the age distributions in the central drop of $\tau_{\text {dep }}$ and the other two groups are $2 \times 10^{-5}$ and 0.07 , while the $p$-value between the flat and rise $\tau_{\text {dep }}$ group is 0.49 . A small $p$-value means the distributions of the two samples are distinct. An AndersonDarling test to those distributions also yields similar results: the $p$-values between the drop $\tau_{\text {center }}$ group and the other two groups are $9 \times 10^{-5}$ and 0.02 , while the $p$-value between the flat and rise groups is 0.61 . This evidence strengthens our suspicion that the centers of the short $\tau_{\text {center }}$ group are currently undergoing a burst of star formation. However, further highresolution data are needed to confirm this hypothesis.

\section{Summary}

We present results from the EDGE survey, a first major, resolved $\mathrm{CO}$ follow-up to an IFU survey of local galaxies (CALIFA). We combine the $\mathrm{CO}$ and optical IFU data to study 

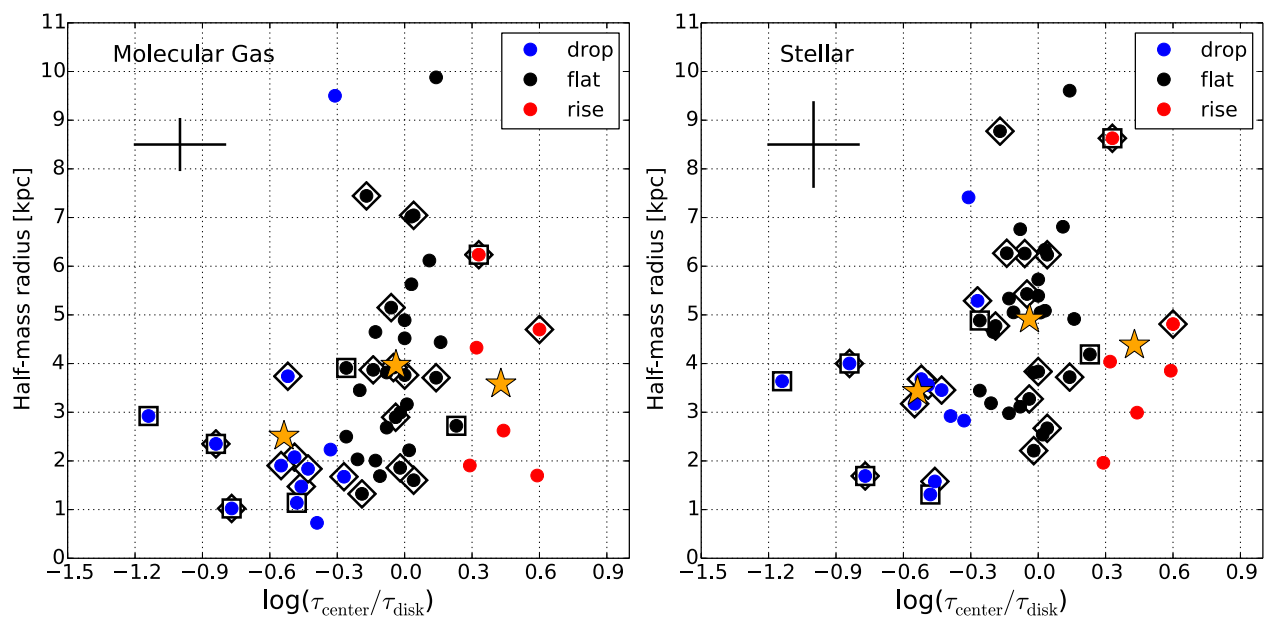

Figure 11. Half-mass-radius of molecular gas (left panel) and stars (right panel) for three groups: central drop (blue dots), flat (black dots), and central rise (red dots) of $\tau_{\text {dep. }}$. The mean values for each of the three groups are marked as orange stars. The typical errors are shown as crosses. The diamond symbols mark the barred galaxies, while the square symbols mark the interacting galaxies. This shows that the molecular gas distribution in the drop $\tau_{\text {center }}$ group and in the disturbed (barred or interacting) galaxies is more compact than that in the other two groups.

Table 1

The Best-fit Parameters from the ODR Method and Correlation Coefficients

\begin{tabular}{|c|c|c|c|c|c|c|c|c|}
\hline \multirow{2}{*}{$\begin{array}{l}x \text {-axis } \\
\text { labels }\end{array}$} & \multirow{2}{*}{$\begin{array}{c}\text { Intercept } \\
a \pm \delta a\end{array}$} & \multirow{2}{*}{$\begin{array}{c}\text { Slope } \\
b \pm \delta b\end{array}$} & \multicolumn{3}{|c|}{ Correlation Coefficient } & \multirow{2}{*}{$\begin{array}{l}p \text {-value } \\
\text { Pearson }\end{array}$} & \multirow{2}{*}{$\begin{array}{c}p \text {-value } \\
\text { Spearman }\end{array}$} & \multirow{2}{*}{$\begin{array}{l}p \text {-value } \\
\text { Kendall }\end{array}$} \\
\hline & & & Pearson & Spearman & $\overline{\text { Kendall }}$ & & & \\
\hline$\overline{\text { SFR }}$ & $0.34 \pm 0.07$ & $-0.54 \pm 0.07$ & -0.68 & -0.64 & -0.48 & $3.81 \times 10^{-8}$ & $3.66 \times 10^{-7}$ & $4.29 \times 10^{-7}$ \\
\hline
\end{tabular}

the variation of $\tau_{\text {dep }}$ between the centers and the disks in 52 local galaxies. Our findings are the following.

1. Contrary to the well-defined value of $\tau_{\text {dep }}$ in galactic disks, galactic centers can have shorter, longer, or similar $\tau_{\text {dep }}$ compared to their disks (Figure 7 ). The short $\tau_{\text {center }}$ group (representing $26.9 \%$ of the samples with $\tau_{\text {center }} \sim 1$ Gyr) resembles the intermediate regime between the disks $\left(\tau_{\text {disk }} \sim 2.4 \mathrm{Gyr}\right)$ and starbursts $\left(\tau_{\text {dep }} \sim 0.2 \mathrm{Gyr}\right)$. Applying the variations of the CO-to- $\mathrm{H}_{2}$ conversion factor (that depends on the total surface density and metallicities) only exaggerates the drop of $\tau_{\text {center }}$.

2. The drop of $\tau_{\text {center }}$ is caused by higher central $\Sigma_{\text {SFR }}$ than those in the disk, not lower $\Sigma_{\text {mol }}$ (Figure 8). Furthermore, galaxies with the higher contrast of stellar surface density in the center (i.e., higher $\Sigma_{*}^{\text {center }} / \Sigma_{*}^{\text {disk }}$ ) tend to have shorter $\tau_{\text {center }} / \tau_{\text {disk }}$. Since the dynamical equilibrium pressure depends on $\Sigma_{*}$ (Blitz \& Rosolowsky 2004, 2006; Ostriker et al. 2010), this suggests that the central drop in $\tau_{\text {dep }}$ is driven by high gas pressure. This is expected for the star-formation self-regulated model, in which the SFR locally adjusts so that feedback from massive stars offsets turbulent energy dissipation and cooling. A high feedback rate (short $\tau_{\text {dep }}$ ) is required to maintain the high pressure in regions where the vertical gravity from stars and gas is very strong (Ostriker et al. 2010; Kim et al. 2011, 2013; Ostriker \& Shetty 2011).

3 . The gradient of oxygen abundance rises toward the center for galaxies in the short $\tau_{\text {center }}$ group, while the gradient is flat in the center of other groups (Figure 9). This could be the stellar mass effect, where the gradient of oxygen abundance is flattening in massive galaxies (as found by
Table 2

The Mean Molecular and Stellar Disk Radius for Each Group

\begin{tabular}{lcccr}
\hline \hline Groups & $\begin{array}{c}R_{1 / 2}^{\text {mol }} \\
\mathrm{kpc}\end{array}$ & $\begin{array}{c}R_{1 / 2}^{*} \\
\mathrm{kpc}\end{array}$ & $\begin{array}{c}\bar{M}_{*} \\
\log \left(M_{\odot}\right)\end{array}$ & $N^{\mathrm{a}}$ \\
\hline Drop & $2.51 \pm 0.58$ & $3.43 \pm 0.41$ & $10.38 \pm 0.11$ & 14 \\
Flat & $3.96 \pm 0.34$ & $4.91 \pm 0.30$ & $10.46 \pm 0.06$ & 32 \\
Rise & $3.58 \pm 0.67$ & $4.38 \pm 0.86$ & $10.47 \pm 0.15$ & 6 \\
\hline Disturbed & $3.21 \pm 0.35$ & $4.29 \pm 0.37$ & $10.54 \pm 0.07$ & 26 \\
Undisturbed & $3.87 \pm 0.45$ & $4.63 \pm 0.35$ & $10.34 \pm 0.07$ & 26 \\
\hline
\end{tabular}

Note. The uncertainty is calculated from the standard deviation divided by the square-root of the number of galaxies.

a The number of galaxies in each group.

Sánchez-Menguiano et al. 2016), or the oxygen abundance is sensitive to the current star-formation efficiency. However, the narrow range of the oxygen abundance variation in our sample $(\sim 0.2 \mathrm{dex})$ becomes the limitation of our analysis.

4. There are two signatures for dynamical effect that drives the variation of $\tau_{\text {center }}$ versus $\tau_{\text {disk }}$. First, the barred and interacting galaxies tend to have lower values of $\log$ ( $\tau_{\text {center }} / \tau_{\text {disk }}$ ) than the unbarred, isolated galaxies (Figure 6). Second, the size of the molecular gas disk is smaller in the drop $\tau_{\text {center }}$ group than in the other groups (Figure 11). We suspect that the bar drives the gas inward toward the center (or in the case of interacting galaxies, the gas loses its angular momentum). This radial gas compression increases the pressure and results in higher star-formation efficiency in the galactic center (Krumholz \& Kruijssen 2015). 
Table 3

The List of Galaxy Properties in the Sample

\begin{tabular}{|c|c|c|c|c|c|c|c|c|c|c|c|c|c|c|c|c|}
\hline No. & Galaxies & $\begin{array}{l}\text { R.A. } \\
\text { h:m:s }\end{array}$ & $\begin{array}{l}\text { Decl. } \\
\text { d:m:s }\end{array}$ & $\begin{array}{r}M_{*}{ }^{\mathrm{a}} \\
\quad \text { lo }\end{array}$ & $\begin{array}{l}M_{\mathrm{mol}}^{\mathrm{b}} \\
\odot)\end{array}$ & $\begin{array}{l}R_{25}{ }^{\mathrm{c}} \\
\mathrm{kpc}\end{array}$ & $\begin{array}{c}\text { Beam }^{\mathrm{d}} \\
\mathrm{kpc}\end{array}$ & $\begin{array}{c}\text { Dist. }^{\mathrm{e}} \\
\text { Mpc }\end{array}$ & $\begin{array}{l}\text { Inc. }^{f} \\
\text { deg. }\end{array}$ & $\begin{array}{l}\text { P.A. }^{\mathrm{f}} \\
\text { deg. }\end{array}$ & $\begin{array}{c}\tau_{\text {center }} \\
\log (\mathrm{yr})\end{array}$ & $\begin{array}{c}\tau_{\text {disk }} \\
\log (\mathrm{yr})\end{array}$ & $\begin{array}{c}\tau_{\text {med }} \\
\log (y r)\end{array}$ & Group & $\overline{\mathrm{Bar}}{ }^{\mathrm{g}}$ & 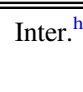 \\
\hline 1 & IC1151 & $15^{\mathrm{h}} 58^{\mathrm{m}} 58^{\mathrm{s}} .5$ & $17^{\circ} 26^{\prime} 26^{\prime \prime} 5$ & 9.82 & 7.93 & 10.01 & 0.67 & 30.80 & 68.0 & 208.9 & 8.94 & 9.04 & 8.99 & flat & $\mathrm{N}$ & $\mathrm{N}$ \\
\hline 2 & IC1199 & $16^{\mathrm{h}} 10^{\mathrm{m}} 10^{\mathrm{s}} 6$ & $10^{\circ} 02^{\prime} 02^{\prime \prime} 4$ & 10.58 & 9.35 & 11.83 & 1.52 & 68.25 & 64.5 & 337.3 & 9.45 & 9.58 & 9.56 & flat & $\mathrm{N}$ & $\mathrm{N}$ \\
\hline 3 & IC1683 & $01^{\mathrm{h}} 22^{\mathrm{m}} 22^{\mathrm{s}} \cdot 6$ & $34^{\circ} 26^{\prime} 26^{\prime \prime} 2$ & 10.56 & 9.68 & 13.34 & 1.47 & 69.73 & 44.8 & 20.6 & 9.15 & 9.64 & 9.64 & drop & $\mathrm{Y}$ & $\mathrm{N}$ \\
\hline 4 & NGC0477 & $01^{\mathrm{h}} 21^{\mathrm{m}} 21^{\mathrm{s}} 3$ & $40^{\circ} 29^{\prime} 29^{\prime \prime} 3$ & 10.70 & 9.54 & 19.29 & 1.86 & 85.42 & 60.0 & 150.0 & 9.37 & 9.68 & 9.66 & drop & $\mathrm{N}$ & $\mathrm{N}$ \\
\hline 5 & NGC0496 & $01^{\mathrm{h}} 23^{\mathrm{m}} 23^{\mathrm{s}} .2$ & $33^{\circ} 31^{\prime} 31^{\prime \prime} 7$ & 10.64 & 9.48 & 11.34 & 1.82 & 87.47 & 57.0 & 38.5 & 9.15 & 9.23 & 9.22 & flat & $\mathrm{N}$ & $\mathrm{N}$ \\
\hline 6 & NGC0551 & $01^{\mathrm{h}} 27^{\mathrm{m}} 27^{\mathrm{s}} \cdot 7$ & $37^{\circ} 10^{\prime} 11^{\prime \prime} 0$ & 10.75 & 9.39 & 16.10 & 1.54 & 74.50 & 64.2 & 320.0 & 9.62 & 9.58 & 9.61 & flat & $\mathrm{Y}$ & $\mathrm{N}$ \\
\hline 7 & NGC2253 & $06^{\mathrm{h}} 43^{\mathrm{m}} 43^{\mathrm{s}} .7$ & $65^{\circ} 12^{\prime} 12^{\prime \prime} 4$ & 10.60 & 9.62 & 10.61 & 1.20 & 51.16 & 47.4 & 300.0 & 9.37 & 9.37 & 9.37 & flat & $\mathrm{Y}$ & $\mathrm{N}$ \\
\hline 8 & NGC2347 & $07^{\mathrm{h}} 16^{\mathrm{m}} 16^{\mathrm{s}} .1$ & $64^{\circ} 42^{\prime} 42^{\prime \prime} 6$ & 10.84 & 9.56 & 15.25 & 1.49 & 63.75 & 50.2 & 189.1 & 9.48 & 9.34 & 9.38 & flat & $\mathrm{Y}$ & $\mathrm{N}$ \\
\hline 9 & NGC2730 & $09^{\mathrm{h}} 02^{\mathrm{m}} 02^{\mathrm{s}} \cdot 3$ & $16^{\circ} 50^{\prime} 50^{\prime \prime} 3$ & 9.93 & 9.00 & 11.52 & 1.26 & 54.78 & 27.7 & 260.8 & 9.13 & 9.24 & 9.23 & flat & $\mathrm{N}$ & $\mathrm{N}$ \\
\hline 10 & NGC2906 & $09^{\mathrm{h}} 32^{\mathrm{m}} 32^{\mathrm{s}} .1$ & $08^{\circ} 26^{\prime} 26^{\prime \prime} 5$ & 10.38 & 9.11 & 7.44 & 0.94 & 37.73 & 55.7 & 265.0 & 9.78 & 9.34 & 9.40 & rise & $\mathrm{N}$ & $\mathrm{N}$ \\
\hline 11 & NGC3381 & $10^{\mathrm{h}} 48^{\mathrm{m}} 48^{\mathrm{s}} .4$ & $34^{\circ} 42^{\prime} 42^{\prime \prime} 7$ & 9.68 & 8.11 & 6.87 & 0.50 & 23.40 & 30.8 & 43.1 & 8.86 & 9.31 & 9.30 & drop & $\mathrm{Y}$ & $\mathrm{N}$ \\
\hline 12 & NGC3811 & $11^{\mathrm{h}} 41^{\mathrm{m}} 41^{\mathrm{s}} 3$ & $47^{\circ} 41^{\prime} 41^{\prime \prime} 4$ & 10.44 & 9.28 & 13.05 & 0.96 & 44.25 & 42.5 & 359.0 & 9.32 & 9.28 & 9.31 & flat & $\mathrm{Y}$ & $\mathrm{N}$ \\
\hline 13 & NGC3815 & $11^{\mathrm{h}} 41^{\mathrm{m}} 41^{\mathrm{s}} 7$ & $24^{\circ} 48^{\prime} 48^{\prime \prime} 0$ & 10.32 & 9.16 & 11.22 & 1.14 & 53.59 & 59.9 & 67.8 & 9.43 & 9.47 & 9.45 & flat & $\mathrm{Y}$ & $\mathrm{N}$ \\
\hline 14 & NGC3994 & $11^{\mathrm{h}} 57^{\mathrm{m}} 57^{\mathrm{s}} \cdot 6$ & $32^{\circ} 16^{\prime} 16^{\prime \prime} 6$ & 10.39 & 9.26 & 5.53 & 1.02 & 44.75 & 59.5 & 188.1 & 9.07 & 8.78 & 8.81 & rise & $\mathrm{N}$ & $\mathrm{N}$ \\
\hline 15 & NGC4047 & $12^{\mathrm{h}} 02^{\mathrm{m}} 02^{\mathrm{s}} .8$ & $48^{\circ} 38^{\prime} 38^{\prime \prime} 2$ & 10.67 & 9.66 & 10.95 & 1.06 & 49.06 & 42.1 & 105.0 & 9.41 & 9.43 & 9.41 & flat & $\mathrm{N}$ & $\mathrm{N}$ \\
\hline 16 & NGC4470 & $12^{\mathrm{h}} 29^{\mathrm{m}} 29^{\mathrm{s}} \cdot 6$ & $07^{\circ} 49^{\prime} 49^{\prime \prime} 4$ & 10.03 & 8.59 & 6.23 & 0.78 & 33.43 & 47.5 & 359.5 & 8.74 & 8.87 & 8.85 & flat & $\mathrm{N}$ & $\mathrm{N}$ \\
\hline 17 & NGC4644 & $12^{\mathrm{h}} 42^{\mathrm{m}} 42^{\mathrm{s}} .7$ & $55^{\circ} 08^{\prime} 08^{\prime \prime} 7$ & 10.48 & 9.20 & 15.77 & 1.60 & 71.65 & 72.9 & 57.0 & 9.59 & 9.56 & 9.57 & flat & $\mathrm{N}$ & $\mathrm{N}$ \\
\hline 18 & NGC4711 & $12^{\mathrm{h}} 48^{\mathrm{m}} 48^{\mathrm{s}} .8$ & $35^{\circ} 19^{\prime} 20^{\prime \prime} 0$ & 10.38 & 9.18 & 10.31 & 1.32 & 58.83 & 58.3 & 215.0 & 9.60 & 9.44 & 9.45 & flat & $\mathrm{N}$ & $\mathrm{N}$ \\
\hline 19 & NGC4961 & $13^{\mathrm{h}} 05^{\mathrm{m}} 05^{\mathrm{s}} .8$ & $27^{\circ} 44^{\prime} 44^{\prime \prime} 0$ & 9.77 & 8.41 & 5.93 & 0.78 & 36.58 & 46.6 & 90.0 & 9.21 & 9.23 & 9.22 & flat & $\mathrm{Y}$ & $\mathrm{N}$ \\
\hline 20 & NGC5000 & $13^{\mathrm{h}} 09^{\mathrm{m}} 09^{\mathrm{s}} .8$ & $28^{\circ} 54^{\prime} 54^{\prime \prime} 4$ & 10.74 & 9.45 & 15.04 & 1.62 & 80.80 & 20.0 & 1.3 & 9.40 & 9.59 & 9.53 & flat & $\mathrm{Y}$ & $\mathrm{N}$ \\
\hline 21 & NGC5016 & $13^{\mathrm{h}} 12^{\mathrm{m}} 12^{\mathrm{s}} .1$ & $24^{\circ} 05^{\prime} 05^{\prime \prime} 7$ & 10.27 & 8.90 & 8.45 & 0.83 & 36.90 & 39.9 & 57.4 & 9.10 & 9.43 & 9.40 & drop & $\mathrm{N}$ & $\mathrm{N}$ \\
\hline 22 & NGC5056 & $13^{\mathrm{h}} 16^{\mathrm{m}} 16^{\mathrm{s}} .2$ & $30^{\circ} 57^{\prime} 57^{\prime \prime} 0$ & 10.64 & 9.45 & 19.14 & 1.96 & 81.14 & 61.4 & 178.0 & 9.03 & 8.43 & 8.51 & rise & $\mathrm{Y}$ & $\mathrm{N}$ \\
\hline 23 & NGC5480 & $14^{\mathrm{h}} 06^{\mathrm{m}} 06^{\mathrm{s}} 4$ & $50^{\circ} 43^{\prime} 43^{\prime \prime} 5$ & 9.97 & 8.92 & 6.57 & 0.52 & 26.96 & 41.5 & 178.0 & 8.99 & 9.20 & 9.20 & flat & $\mathrm{N}$ & $\mathrm{N}$ \\
\hline 24 & NGC5520 & $14^{\mathrm{h}} 12^{\mathrm{m}} 12^{\mathrm{s}} 4$ & $50^{\circ} 20^{\prime} 20^{\prime \prime} 9$ & 9.87 & 8.67 & 6.25 & 0.55 & 26.73 & 59.1 & 245.1 & 8.99 & 9.45 & 9.30 & drop & $\mathrm{Y}$ & $\mathrm{N}$ \\
\hline 25 & NGC5633 & $14^{\mathrm{h}} 27^{\mathrm{m}} 27^{\mathrm{s}} .5$ & $46^{\circ} 08^{\prime} 08^{\prime \prime} 8$ & 10.20 & 9.14 & 5.29 & 0.71 & 33.38 & 41.9 & 16.9 & 9.25 & 9.23 & 9.24 & flat & $\mathrm{N}$ & $\mathrm{N}$ \\
\hline 26 & NGC5657 & $14^{\mathrm{h}} 30^{\mathrm{m}} 30^{\mathrm{s}} .7$ & $29^{\circ} 10^{\prime} 10^{\prime \prime} 8$ & 10.29 & 9.11 & 14.34 & 1.20 & 56.33 & 68.3 & 344.0 & 9.00 & 9.55 & 9.52 & drop & $\mathrm{Y}$ & $\mathrm{N}$ \\
\hline 27 & NGC5732 & $14^{\mathrm{h}} 40^{\mathrm{m}} 40^{\mathrm{s}} .7$ & $38^{\circ} 38^{\prime} 38^{\prime \prime} 3$ & 10.03 & 8.82 & 9.66 & 1.25 & 54.00 & 58.4 & 43.2 & 9.16 & 9.42 & 9.41 & flat & $\mathrm{N}$ & $\mathrm{N}$ \\
\hline 28 & NGC5784 & $14^{\mathrm{h}} 54^{\mathrm{m}} 54^{\mathrm{s}} 3$ & $42^{\circ} 33^{\prime} 33^{\prime \prime} 5$ & 11.09 & 9.40 & 17.12 & 1.67 & 79.42 & 45.0 & 252.0 & 9.26 & 10.40 & 9.95 & drop & $\mathrm{N}$ & $\mathrm{Y}$ \\
\hline 29 & NGC5930 & $15^{\mathrm{h}} 26^{\mathrm{m}} 26^{\mathrm{s}} .1$ & $41^{\circ} 40^{\prime} 40^{\prime \prime} 6$ & 10.40 & 9.33 & 10.01 & 0.83 & 37.23 & 45.0 & 155.0 & 9.27 & 10.04 & 9.71 & drop & $\mathrm{Y}$ & $\mathrm{Y}$ \\
\hline 30 & NGC5934 & $15^{\mathrm{h}} 28^{\mathrm{m}} 28^{\mathrm{s}} .2$ & $42^{\circ} 55^{\prime} 55^{\prime \prime} 8$ & 10.66 & 9.81 & 7.35 & 1.76 & 82.71 & 55.0 & 5.0 & 10.00 & 9.77 & 9.79 & flat & $\mathrm{N}$ & $\mathrm{Y}$ \\
\hline 31 & NGC5947 & $15^{\mathrm{h}} 30^{\mathrm{m}} 30^{\mathrm{s}} .6$ & $42^{\circ} 43^{\prime} 43^{\prime \prime} 0$ & 10.67 & 9.26 & 14.61 & 1.92 & 86.07 & 32.2 & 206.6 & 9.09 & 9.61 & 9.59 & drop & $\mathrm{Y}$ & $\mathrm{N}$ \\
\hline 32 & NGC5953 & $15^{\mathrm{h}} 34^{\mathrm{m}} 34^{\mathrm{s}} .5$ & $15^{\circ} 11^{\prime} 11^{\prime \prime} 6$ & 10.18 & 9.49 & 6.09 & 0.61 & 28.43 & 26.1 & 43.3 & 9.12 & 9.60 & 9.47 & drop & $\mathrm{N}$ & $\mathrm{Y}$ \\
\hline 33 & NGC5980 & $15^{\mathrm{h}} 41^{\mathrm{m}} 41^{\mathrm{s}} .5$ & $15^{\circ} 47^{\prime} 47^{\prime \prime} 3$ & 10.61 & 9.70 & 14.10 & 1.27 & 59.36 & 66.2 & 15.0 & 9.47 & 9.15 & 9.19 & rise & $\mathrm{N}$ & $\mathrm{N}$ \\
\hline 34 & NGC6004 & $15^{\mathrm{h}} 50^{\mathrm{m}} 50^{\mathrm{s}} .4$ & $18^{\circ} 56^{\prime} 56^{\prime \prime} 4$ & 10.66 & 9.33 & 15.19 & 1.22 & 55.21 & 37.3 & 277.3 & 9.61 & 9.66 & 9.63 & flat & $\mathrm{Y}$ & $\mathrm{N}$ \\
\hline 35 & NGC6060 & $16^{\mathrm{h}} 05^{\mathrm{m}} 05^{\mathrm{s}} .9$ & $21^{\circ} 29^{\prime} 29^{\prime \prime}{ }^{\prime} 1$ & 10.78 & 9.68 & 17.41 & 1.28 & 63.24 & 64.3 & 102.0 & 9.39 & 9.36 & 9.38 & flat & $\mathrm{N}$ & $\mathrm{N}$ \\
\hline 36 & NGC6155 & $16^{\mathrm{h}} 26^{\mathrm{m}} 26^{\mathrm{s}} .1$ & $48^{\circ} 22^{\prime} 22^{\prime \prime} 0$ & 10.18 & 8.94 & 6.68 & 0.77 & 34.60 & 44.7 & 130.0 & 9.02 & 9.10 & 9.08 & flat & $\mathrm{N}$ & $\mathrm{N}$ \\
\hline 37 & NGC6186 & $16^{\mathrm{h}} 34^{\mathrm{m}} 34^{\mathrm{s}} .4$ & $21^{\circ} 32^{\prime} 32^{\prime \prime} 5$ & 10.41 & 9.46 & 9.68 & 0.92 & 42.38 & 71.2 & 69.8 & 9.32 & 9.49 & 9.46 & flat & $\mathrm{Y}$ & $\mathrm{N}$ \\
\hline 38 & NGC6301 & $17^{\mathrm{h}} 08^{\mathrm{m}} 08^{\mathrm{s}} .5$ & $42^{\circ} 20^{\prime} 20^{\prime \prime} 3$ & 10.98 & 9.96 & 31.24 & 2.63 & 121.36 & 52.8 & 288.5 & 9.75 & 9.61 & 9.65 & flat & $\mathrm{N}$ & $\mathrm{N}$ \\
\hline 39 & NGC7738 & $23^{\mathrm{h}} 44^{\mathrm{m}} 44^{\mathrm{s}} .0$ & $00^{\circ} 31^{\prime} 31^{\prime \prime} 0$ & 11.01 & 9.99 & 16.87 & 1.90 & 97.82 & 65.6 & 244.7 & 9.17 & 10.01 & 9.74 & drop & $\mathrm{Y}$ & $\mathrm{Y}$ \\
\hline 40 & NGC7819 & $00^{\mathrm{h}} 04^{\mathrm{m}} 04^{\mathrm{s}} .4$ & $31^{\circ} 28^{\prime} 28^{\prime \prime} 3$ & 10.41 & 9.27 & 14.99 & 1.43 & 71.62 & 54.0 & 280.3 & 9.28 & 9.55 & 9.54 & drop & $\mathrm{Y}$ & $\mathrm{N}$ \\
\hline 41 & UGC03253 & $05^{\mathrm{h}} 19^{\mathrm{m}} 19^{\mathrm{s}} .7$ & $84^{\circ} 03^{\prime} 03^{\prime \prime} 1$ & 10.43 & 8.88 & 11.88 & 1.57 & 59.46 & 58.3 & 267.7 & 8.88 & 9.31 & 9.29 & drop & $\mathrm{Y}$ & $\mathrm{N}$ \\
\hline 42 & UGC04132 & $07^{\mathrm{h}} 59^{\mathrm{m}} 59^{\mathrm{s}} .2$ & $32^{\circ} 54^{\prime} 54^{\prime \prime} 9$ & 10.74 & 10.02 & 13.51 & 1.70 & 75.35 & 72.0 & 212.6 & 9.35 & 9.41 & 9.41 & flat & $\mathrm{Y}$ & $\mathrm{N}$ \\
\hline 43 & UGC04461 & $08^{\mathrm{h}} 33^{\mathrm{m}} 33^{\mathrm{s}} .4$ & $52^{\circ} 31^{\prime} 31^{\prime \prime} 9$ & 10.17 & 9.24 & 14.51 & 1.59 & 72.27 & 70.1 & 215.8 & 9.36 & 9.35 & 9.36 & flat & $\mathrm{N}$ & $\mathrm{N}$ \\
\hline 44 & UGC05108 & $09^{\mathrm{h}} 35^{\mathrm{m}} 35^{\mathrm{s}} .4$ & $29^{\circ} 48^{\prime} 48^{\prime \prime} 8$ & 10.90 & 9.75 & 18.84 & 2.81 & 118.41 & 66.1 & 133.1 & 9.47 & 9.61 & 9.53 & flat & $\mathrm{Y}$ & $\mathrm{N}$ \\
\hline 45 & UGC07012 & $12^{\mathrm{h}} 02^{\mathrm{m}} 02^{\mathrm{s}} .1$ & $29^{\circ} 50^{\prime} 50^{\prime \prime} 9$ & 9.70 & 8.35 & 6.96 & 0.92 & 44.28 & 60.5 & 182.1 & 8.75 & 9.14 & 9.13 & drop & $\mathrm{N}$ & $\mathrm{N}$ \\
\hline 46 & UGC08107 & $12^{\mathrm{h}} 59^{\mathrm{m}} 59^{\mathrm{s}} .7$ & $53^{\circ} 20^{\prime} 20^{\prime \prime} 5$ & 11.00 & 10.11 & 40.43 & 2.75 & 121.62 & 71.4 & 233.2 & 9.92 & 9.59 & 9.60 & rise & $\mathrm{Y}$ & $\mathrm{Y}$ \\
\hline 47 & UGC09067 & $14^{\mathrm{h}} 10^{\mathrm{m}} 10^{\mathrm{s}} .8$ & $15^{\circ} 12^{\prime} 12^{\prime \prime} 6$ & 10.76 & 9.83 & 13.54 & 2.75 & 114.50 & 62.4 & 14.6 & 9.46 & 9.46 & 9.46 & flat & $\mathrm{N}$ & $\mathrm{N}$ \\
\hline
\end{tabular}


Table 3

(Continued)

\begin{tabular}{|c|c|c|c|c|c|c|c|c|c|c|c|c|c|c|c|c|}
\hline No. & Galaxies & $\begin{array}{l}\text { R.A. } \\
\text { h:m:s }\end{array}$ & $\begin{array}{l}\text { Decl. } \\
\text { d:m:s }\end{array}$ & $\begin{array}{r}M_{*}{ }^{\mathrm{a}} \\
\quad \text { lo }\end{array}$ & $\begin{array}{l}M_{\mathrm{mol}}^{\mathrm{b}} \\
\odot)\end{array}$ & $\begin{array}{l}R_{25}{ }^{\mathrm{c}} \\
\mathrm{kpc}\end{array}$ & $\begin{array}{c}\text { Beam }^{\mathrm{d}} \\
\mathrm{kpc}\end{array}$ & $\begin{array}{l}\text { Dist. }^{\mathrm{e}} \\
\mathrm{Mpc}\end{array}$ & $\begin{array}{l}\text { Inc. }{ }^{\mathrm{f}} \\
\text { deg. }\end{array}$ & $\begin{array}{l}\text { P.A. }^{f} \\
\text { deg. }\end{array}$ & $\begin{array}{c}\tau_{\text {center }} \\
\log (\mathrm{yr})\end{array}$ & $\begin{array}{c}\tau_{\text {disk }} \\
\log (\mathrm{yr})\end{array}$ & $\begin{array}{c}\tau_{\text {med }} \\
\log (\mathrm{yr})\end{array}$ & Group & $\mathrm{Bar}^{\mathrm{g}}$ & Inter. $^{\mathrm{h}}$ \\
\hline 48 & UGC09476 & $14^{\mathrm{h}} 41^{\mathrm{m}} 41^{\mathrm{s}} .5$ & $44^{\circ} 30^{\prime} 30^{\prime \prime} 8$ & 10.23 & 9.15 & 10.19 & 1.01 & 46.63 & 48.5 & 312.0 & 9.32 & 9.52 & 9.50 & flat & $\mathrm{N}$ & $\mathrm{N}$ \\
\hline 49 & UGC09542 & $14^{\mathrm{h}} 49^{\mathrm{m}} 49^{\mathrm{s}} \cdot 0$ & $42^{\circ} 27^{\prime} 27^{\prime \prime} 8$ & 10.32 & 9.31 & 16.64 & 1.65 & 79.70 & 72.7 & 214.3 & 9.56 & 9.56 & 9.56 & flat & $\mathrm{N}$ & $\mathrm{N}$ \\
\hline 50 & UGC09759 & $15^{\mathrm{h}} 10^{\mathrm{m}} 10^{\mathrm{s}} \cdot 7$ & $55^{\circ} 21^{\prime} 21^{\prime \prime} 0$ & 9.81 & 9.07 & 9.55 & 1.03 & 49.25 & 66.8 & 54.7 & 10.20 & 9.61 & 9.69 & rise & $\mathrm{N}$ & $\mathrm{N}$ \\
\hline 51 & UGC10205 & $16^{\mathrm{h}} 06^{\mathrm{m}} 06^{\mathrm{s}} .7$ & $30^{\circ} 05^{\prime} 05^{\prime \prime} 9$ & 10.88 & 9.60 & 19.95 & 2.21 & 94.92 & 51.7 & 118.6 & 9.56 & 9.82 & 9.81 & flat & $\mathrm{N}$ & Y \\
\hline 52 & UGC10710 & $17^{\mathrm{h}} 06^{\mathrm{m}} 06^{\mathrm{s}} .9$ & $43^{\circ} 07^{\prime} 07^{\prime \prime} 3$ & 10.72 & 9.88 & 29.51 & 2.63 & 121.69 & 69.6 & 329.5 & 9.61 & 9.50 & 9.50 & flat & $\mathrm{N}$ & $\mathrm{N}$ \\
\hline
\end{tabular}

Notes.

$\sim \quad{ }^{2}$ The stellar mass assuming Kroupa IMF from the CALIFA survey (Sánchez et al. 2016).

${ }^{\mathrm{b}}$ The molecular gas mass assuming a CO-to- $\mathrm{H}_{2}$ conversion factor of $4.4 M_{\odot} \mathrm{pc}^{-2}\left(\mathrm{~K} \mathrm{~km} \mathrm{~s}^{-1} \mathrm{pc}^{2}\right)^{-1}$ from the EDGE survey (Bolatto et al. 2017), including mass contribution from helium.

${ }^{c}$ The radius where the surface brightness is $25 \mathrm{mag} \mathrm{arcsec}^{-2}$ in the $B$-band, from the HyperLEDA catalog (Makarov et al. 2014).

${ }^{\mathrm{d}}$ The physical beam size, calculated from the geometric mean of the major and minor axes of the EDGE beam.

e The luminosity distance computed from the CALIFA redshift for ionized gas lines assuming $H_{0}=70 \mathrm{~km} \mathrm{~s}^{-1}, \Omega_{\mathrm{m}}=0.27$, and $\Omega_{\Lambda}=0.73$.

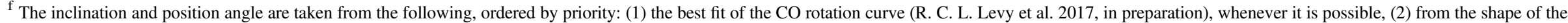
outer isophote, or (3) from the HyperLEDA catalog (Makarov et al. 2014).

${ }^{g}$ The bar assignments (Yes or No) are taken from the following, ordered by priority: (1) the photometric fit from Méndez-Abreu et al. (2017), or (2) the HyperLEDA catalog (Makarov et al. 2014)

${ }^{\mathrm{h}}$ The assignment for interacting galaxies (Yes or No), taken from Barrera-Ballesteros et al. (2015). 

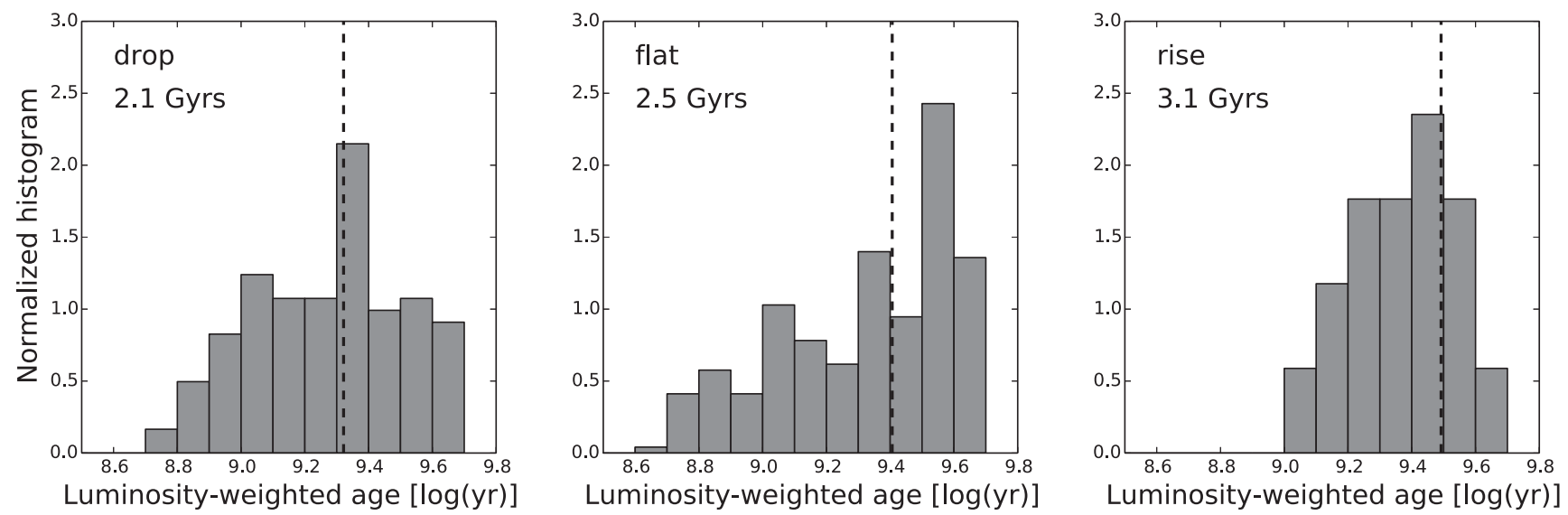

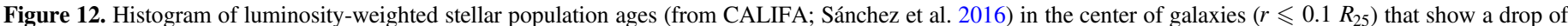

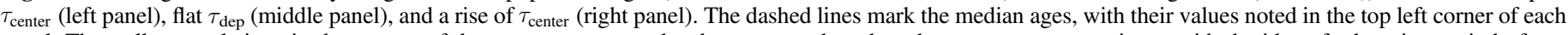

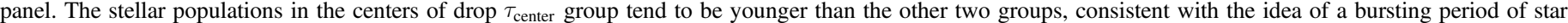
formation.

In conclusion, these findings imply that the formation of stars from the molecular gas depends on the local environment within a galaxy (such as $\Sigma_{*}$ ) and the galaxy dynamics induced by bar or interactions. In the future, we are interested to measure the dense gas (as traced by HCN lines) to investigate whether the short $\tau_{\text {center }}$ is also due to a higher fraction of the dense gas in the center. In addition, measuring the shear rate and the inflow speed in barred galaxies will give better evidence of the importance of galactic dynamics in driving $\tau_{\text {dep. }}$. Finally, expanding our sample toward early-type and lowmass galaxies using ALMA is a natural approach to expand our statistical sample in the three groups of $\tau_{\text {dep. }}$.

We thank the referee, Christine Wilson, for her valuable input that greatly improved the manuscript. We also thank John Carpenter for his help in managing the schedule of CARMA observations and Christopher McKee for insightful discussion.

The works of D.U. and L.B. are supported by the National Science Foundation (NSF) under grants AST-1140063 and AST-1616924. A.D.B. and R.C.L. acknowledge support from NSF through grants AST-1412419 and AST-1615960. A.D.B. also acknowledges visiting support by the Alexander von Humboldt Foundation. T.W. and Y.C. acknowledge support from NSF through grants AST-1139950 and AST-1616199. The work of ECO is supported by the NSF under grant AST1312006. S.F.S. acknowledges the PAPIIT-DGAPA-IA101217 project and CONACYT-IA-180125. R.G.B. acknowledges support through grant AYA2016-77846-P. E.R. is supported by a Discovery Grant from NSERC of Canada. S.V. acknowledges support from NSF AST-1615960.

We acknowledge the usage of the HyperLeda database (http://leda.univ-lyon1.fr). Support for the CARMA construction was derived from the states of California, Illinois, and Maryland, the James S. McDonnell Foundation, the Gordon and Betty Moore Foundation, the Kenneth T. and Eileen L. Norris Foundation, the University of Chicago, the Associates of the California Institute of Technology, and NSF. This research is based on observations collected at the Centro Astronomico Hispano Aleman (CAHA) at Calar Alto, operated jointly by the Max-Planck Institute for Astronomy (MPIA) and the Instituto de Astrofisica de Andalucia (CSIC).

Software: Pipe3D (version 2.2; Sánchez et al. 2016), MIRIAD (Sault et al. 1995), idl_mommaps.pro (Wong et al.
2013), linmix_err.pro (Kelly 2007), matplotlib (Hunter 2007), and SciPy (Jones et al. 2001).

\section{Appendix A \\ List of Galaxy Properties in the Sample}

In Table 3, we list the properties of galaxies in our sample. Note that this list only contains 52 galaxies out of 126 galaxies in the full EDGE sample. For a complete list of EDGE sample, see Bolatto et al. (2017).

\section{Appendix B The Effect of Nondetections}

The classification of $\tau_{\text {center }}$ in Section 4.2 only takes into account the detected regions in both $\Sigma_{\text {mol }}$ and $\Sigma_{\text {SFR }}$ (shown as gray circles in Figure 13). We now check the robustness of our results by including the upper and lower limits of $\tau_{\text {dep }}$. For the upper limit of $\tau_{\text {dep }}, \Sigma_{\text {mol }}$ is nondetected and is replaced by $1 \sigma_{\text {rms }}$, while $\Sigma_{\mathrm{SFR}}$ is detected. Conversely, for the lower limit of $\tau_{\text {dep }}, \Sigma_{\text {mol }}$ is detected, while $\Sigma_{\mathrm{SFR}}$ is notdetected and is replaced by $1 \sigma_{\mathrm{rms}}$. The upper and lower limits of $\tau_{\text {dep }}$ are shown as triangles pointing down and up, respectively, in Figure 13. Then, we calculate the median value of $\tau_{\text {dep }}$ (after the inclusion of upper and lower limits) in each radial bin (shown as the blue lines in Figure 13). As a comparison, the median values of $\tau_{\text {dep }}$ using only the detected regions in radial bins are shown as the black lines. The upper limits tend to have lower $\tau_{\text {dep }}$ than that in detected regions. Therefore, the blue line can be lower than the black line where upper limits are dominant (as in NGC2480 and NGC5520). An inverse situation happens where lower limits are dominant (as in NGC3811). If detected regions are dominant then the blue and black lines are coincidence with each other (as in NGC5633 and NGC2906).

As in Section 4.2, we define $\tau_{\text {center }}$ as the median of $\tau_{\text {dep }}$ within $0.1 R_{25}$ and $\tau_{\text {disk }}$ as the median of $\tau_{\text {dep }}$ between 0.1 and $0.7 R_{25}$. Then, we compare the value of $\tau_{\text {center }}$ and $\tau_{\text {disk }}$ by using a threshold value of 0.26 dex. If $\log \left(\tau_{\text {center }} / \tau_{\text {disk }}\right)$ is less than -0.26 , then that galaxy is in the drop category, and vice versa. For $\log \left(\tau_{\text {center }} / \tau_{\text {disk }}\right)$ in between -0.26 dex and 0.26 dex, we assign that galaxy in the flat category.

In Figure 14, we plot the values of $\log \left(\tau_{\text {center }} / \tau_{\text {disk }}\right)$ that are obtained in Section 4.2 as the $x$-axis and by including nondetection as the $y$-axis. The relationship between the two 

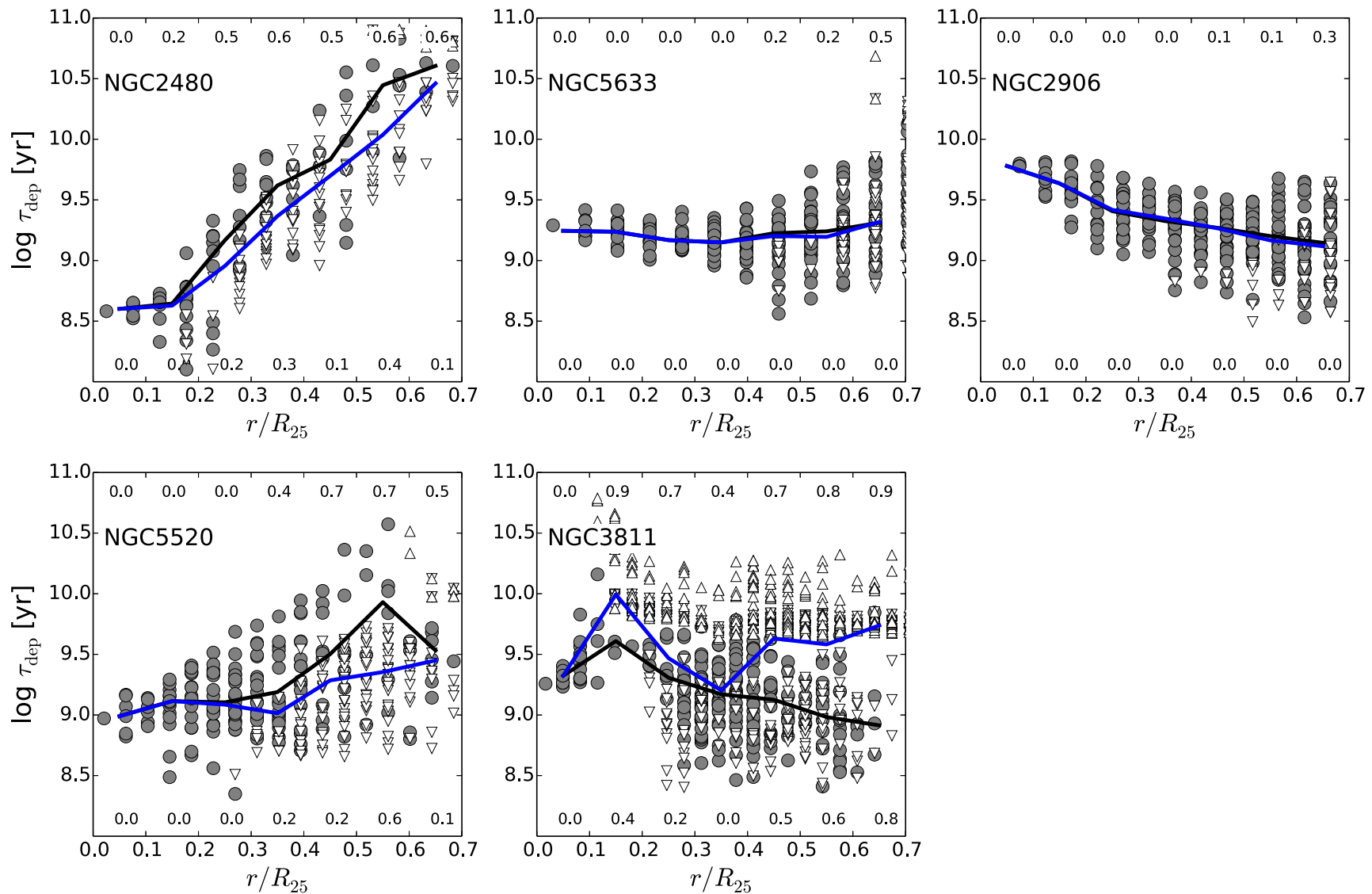

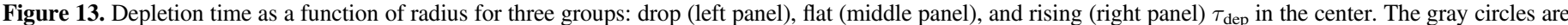

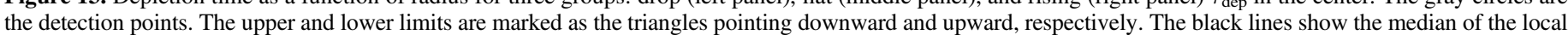

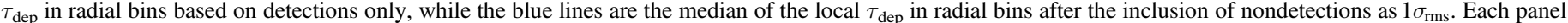

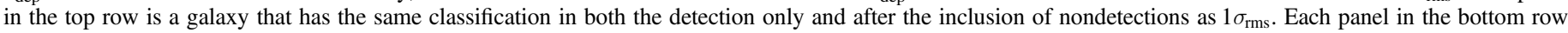

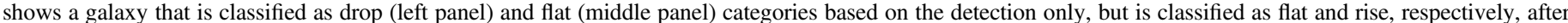

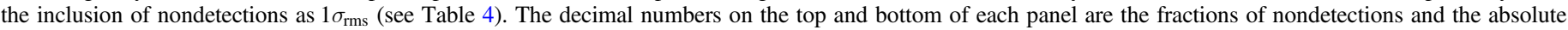
difference (in dex) between the black and blue lines, respectively.

Table 4

Comparisons of Categories between the Method in Section 4.2 (Detection Only) and Azimuthal-average Profile by Including Non-detections (This Appendix)

\begin{tabular}{|c|c|c|c|c|c|}
\hline \multirow{2}{*}{\multicolumn{2}{|c|}{$\begin{array}{c}\text { Include Nondetec- } \\
\text { tions as }\end{array}$}} & \multicolumn{4}{|c|}{ Detection Only } \\
\hline & & \multirow{2}{*}{$\frac{\text { Drop }}{10}$} & \multirow{2}{*}{$\frac{\text { Flat }}{2}$} & \multirow{2}{*}{$\frac{\text { Rise }}{0}$} & \multirow{2}{*}{$\frac{\text { Total }}{12}$} \\
\hline $1 \sigma_{\mathrm{rms}}$ & Drop & & & & \\
\hline & Flat & 4 & 30 & 0 & 34 \\
\hline & Rise & 0 & 0 & 6 & 6 \\
\hline & Total & 14 & 32 & 6 & 52 \\
\hline \multirow[t]{4}{*}{$2 \sigma_{\mathrm{rms}}$} & Drop & 13 & 7 & 0 & 20 \\
\hline & Flat & 1 & 25 & 2 & 28 \\
\hline & Rise & 0 & 0 & 4 & 4 \\
\hline & Total & 14 & 32 & 6 & 52 \\
\hline
\end{tabular}

values is close to a one-to-one relation (black line). This means that the inclusion of nondetections almost does not change the results of our analysis in the main text.

Another way to see the effect on nondetections is by comparing the number of galaxies in each group, obtained with the detections only and including the nondetections (as summarizes in Table 4). For detections only, there are 14 galaxies in the drop $\tau_{\text {center }}$ group. After the inclusion of nondetections as $1 \sigma_{\text {rms }}$, only 10 of them stay in the drop $\tau_{\text {center }}$ group, while 4 of them are categorized as the flat $\tau_{\text {dep }}$ group. Furthermore, from 32 galaxies in the flat $\tau_{\text {dep }}$ group analyzed using detections only, 30 of them stay in the flat $\tau_{\text {dep }}$ group after the inclusion of non-detections as $1 \sigma_{\text {rms }}$, while 2 of them are categorized as the drop $\tau_{\text {center }}$ group. On the other hand, the number of galaxies in the rising $\tau_{\text {center }}$ group is not affected by the inclusion of nondetections as $1 \sigma_{\text {rms. }}$. In total, there are $10+2=12$ galaxies in the drop $\tau_{\text {center }}$ group, $30+4=34$ galaxies in the flat $\tau_{\text {dep }}$ group, and 6 galaxies in the rising $\tau_{\text {center }}$ group after the inclusion of nondetections as $1 \sigma_{\mathrm{rms}}$. The numbers of galaxies that stay in the same group are located in the diagonal of Table 4, i.e., $10+30+6=46$ galaxies. If we to refer this as "true-positive," then we get a true-positive rate of $46 / 52=88.5 \%$, where 52 is the number of galaxies in our sample. For completeness, we also do the same analysis by replacing non-detections with $2 \sigma_{\text {rms }}$ (Table 4$)$. In this case, the true positive rate reduces to $80.8 \%$.

\section{Appendix C The Effect of Physical Resolutions}

The measurement of $\tau_{\text {dep }}$ is known to be scale dependent, that is, the value of $\tau_{\text {dep }}$ changes as a function of physical scale. This difference can be due to the evolutionary effect of star- 


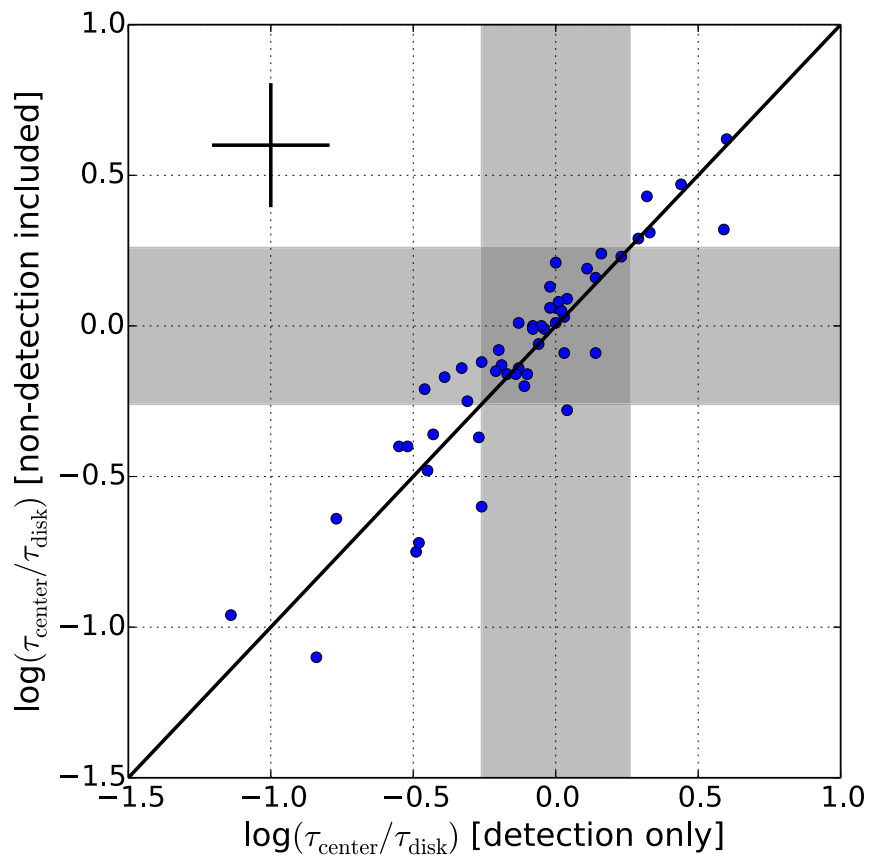

Figure 14. Comparison of $\log \left(\tau_{\text {center }} / \tau_{\text {disk }}\right)$ between the detection only and after including non-detection as $1 \sigma_{\text {rms. }}$. The typical uncertainty is about 0.2 dex (the cross sign). The black line is a one-to-one correlation. The gray bands mark the spaces of the flat category in each method. The number of galaxies in each category is tabulated in Table 4.

forming regions at scale $\lesssim 0.5 \mathrm{kpc}$, where the peaks of $\mathrm{CO}$ emission and SFR do not coincide with each other (Kennicutt et al. 2007; Schruba et al. 2010; Kruijssen \& Longmore 2014). By using simple models, Calzetti et al. (2012) found that the scale dependence of $\tau_{\text {dep }}$ is also due to the stochastic sampling of molecular cloud mass functions. However, there is a general trend that $\tau_{\text {dep }}$ reaches an approximately constant value at scales larger than 1-2 kpc. Interestingly, the central drop of $\tau_{\text {dep }}$ that was reported by Leroy et al. (2013) occurred at radius $<1 \mathrm{kpc}$. Does the central drop of $\tau_{\text {dep }}$ still exists at scales larger than $1 \mathrm{kpc}$ ?

To test the scale dependence of $\tau_{\text {dep }}$, we degrade the physical resolution of galaxies into five scales, from $1 \mathrm{kpc}$ to $3 \mathrm{kpc}$ with an increment of $0.5 \mathrm{kpc}$. Only galaxies with native resolutions smaller than a given degraded resolution are included. For example, a galaxy with a native resolution of $0.7 \mathrm{kpc}$ is included in all resolution bins, while a galaxy with a native resolution of $2.2 \mathrm{kpc}$ is only included in degraded resolutions of 2.5 and $3 \mathrm{kpc}$. In this case, the numbers of galaxies increases from smaller to larger degraded physical resolutions.

The process to make a common physical resolution between galaxies is described below. First, we deproject the EDGECALIFA maps of each galaxy by stretching it through its minor axis using an IDL function, GAL_FLAT. During this step, the surface brightness of galaxies is corrected for inclination. Then, we convolve each map to a common physical resolution, corresponding to each degraded resolution, using an IDL function, SMOOTH3D. Finally, we resample each map using a MIRIAD task, REGRID, so that each resolution element contains approximately 4 pixels.

In Figure 15, we show $\log \left(\tau_{\text {center }} / \tau_{\text {disk }}\right)$ of each galaxy at various common physical resolution as blue dots. The data points at resolutions smaller than $1 \mathrm{kpc}$ are the values at their

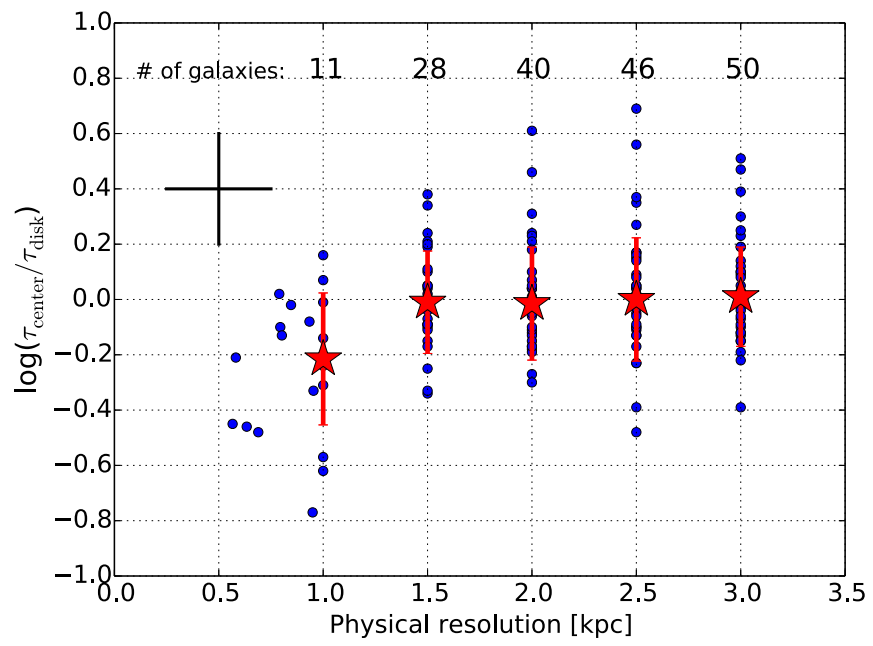

Figure 15. Values of $\tau_{\text {center }}$ relative to $\tau_{\text {disk }}$ are plotted against the common physical resolution (blue dots). The red stars are the median values in each physical resolution. The typical uncertainty is marked as a cross. The numbers of galaxies in each resolution bin are indicated at the top of the figure. This figure shows that the central drop of $\tau_{\text {dep }}$ is scale dependent and more prominent at scales $\lesssim 1 \mathrm{kpc}$.

native resolution that are included in Figure 15 as comparisons. The red stars mark the median values of $\log \left(\tau_{\text {center }} / \tau_{\text {disk }}\right)$ at each resolution. Interestingly, the central drop of $\tau_{\text {dep }}$ is more prominent at resolution $\lesssim 1 \mathrm{kpc}$. While there are scatters in each resolution bin, the median values of $\log \left(\tau_{\text {center }} / \tau_{\text {disk }}\right)$ are approximately zero at resolutions larger than $1 \mathrm{kpc}$. This confirms that the relative values of $\tau_{\text {center }}$ with respect to $\tau_{\text {disk }}$ are indeed scale dependent, and the physical origin of the central drop of $\tau_{\text {dep }}$ is beyond the scale of our data resolution.

If we consider that the galaxies with central drops of $\tau_{\text {dep }}$ undergo a nuclear burst of star formation, this implies that the size of that burst is smaller than $1 \mathrm{kpc}$ within the galactic center. A dynamical model of the Milky Way from Krumholz \& Kruijssen (2015) predicts that the gravitational instability occurs at a scale of $\sim 100 \mathrm{pc}$ in the center. This instability is the result of gas accumulation in the center, driven by the inflow motion due to bar dynamics. Within a 17 Myr timescale, this gravitational instability leads to a burst of star formation that sweeps out the gas, and then the gas accumulation process restarts again. In this view, our data give tentative evidence that a burst of star formation may happen in galactic centers.

\section{ORCID iDs}

Dyas Utomo (1D https://orcid.org/0000-0003-4161-2639

Alberto D. Bolatto (10 https://orcid.org/0000-0002-5480-5686

Tony Wong (ib https://orcid.org/0000-0002-7759-0585

Eve C. Ostriker (i) https://orcid.org/0000-0002-0509-9113

Leo Blitz (iD https://orcid.org/0000-0002-4272-4432

Sebastian F. Sanchez (iD https://orcid.org/0000-00016444-9307

Adam K. Leroy (iD https://orcid.org/0000-0002-2545-1700

Yixian Cao (i) https://orcid.org/0000-0001-5301-1326

Helmut Dannerbauer (i) https://orcid.org/0000-0001-

7147-3575

Ruben Garcia-Benito (iD https://orcid.org/0000-00027077-308X

Bernd Husemann (iD https://orcid.org/0000-0003-2901-6842

Damian Mast (1) https://orcid.org/0000-0003-0469-3193

Erik Rosolowsky (i) https://orcid.org/0000-0002-5204-2259 


\section{References}

Abazajian, K. N., Adelman-McCarthy, J. K., Agüeros, M. A., et al. 2009, ApJS, 182, 543

Allende Prieto, C., Lambert, D. L., \& Asplund, M. 2001, ApJL, 556, L63

Alloin, D., Collin-Souffrin, S., Joly, M., \& Vigroux, L. 1979, A\&A, 78, 200

Baldwin, J. A., Phillips, M. M., \& Terlevich, R. 1981, PASP, 93, 5

Barrera-Ballesteros, J. K., García-Lorenzo, B., Falcón-Barroso, J., et al. 2015, A\&A, 582, A21

Bauermeister, A., Blitz, L., \& Ma, C.-P. 2010, ApJ, 717, 323

Belfiore, F., Maiolino, R., Maraston, C., et al. 2016, MNRAS, 461, 3111

Bigiel, F., Leroy, A., Walter, F., et al. 2008, AJ, 136, 2846

Bigiel, F., Leroy, A. K., Walter, F., et al. 2011, ApJL, 730, L13

Blitz, L., \& Rosolowsky, E. 2004, ApJL, 612, L29

Blitz, L., \& Rosolowsky, E. 2006, ApJ, 650, 933

Bock, D. C.-J., Bolatto, A. D., Hawkins, D. W., et al. 2006, Proc. SPIE, 6267, 626713

Boggs, P. T., Byrd, R. H., \& Schnabel, R. B. 1987, SIAM J. Sci. and Stat. Comput., 8, 1052

Bolatto, A. D., Leroy, A. K., Jameson, K., et al. 2011, ApJ, 741, 12

Bolatto, A. D., Leroy, A. K., Rosolowsky, E., Walter, F., \& Blitz, L. 2008, ApJ, 686, 948

Bolatto, A. D., Wolfire, M., \& Leroy, A. K. 2013, ARA\&A, 51, 207

Bolatto, A. D., Wong, T., Utomo, D., et al. 2017, ApJ, 846, 159

Calzetti, D., Armus, L., Bohlin, R. C., et al. 2000, ApJ, 533, 682

Calzetti, D., Kennicutt, R. C., Engelbracht, C. W., et al. 2007, ApJ, 666, 870

Calzetti, D., Liu, G., \& Koda, J. 2012, ApJ, 752, 98

Cardelli, J. A., Clayton, G. C., \& Mathis, J. S. 1989, ApJ, 345, 245

Catalán-Torrecilla, C., Gil de Paz, A., Castillo-Morales, A., et al. 2015, A\&A, 584, A87

Daddi, E., Elbaz, D., Walter, F., et al. 2010, ApJL, 714, L118

de Vaucouleurs, G., de Vaucouleurs, A., Corwin, H. G., Jr., et al. 1991, Third Reference Catalogue of Bright Galaxies (New York: Springer)

Domínguez, A., Siana, B., Henry, A. L., et al. 2013, ApJ, 763, 145

Elmegreen, B. G. 1997, RMxAA, 6, 165

Falcón-Barroso, J., Sánchez-Blázquez, P., Vazdekis, A., et al. 2011, A\&A, 532, A95

Feigelson, E. D., \& Babu, G. J. 2013, in Planets, Stars and Stellar Systems, ed. T. D. Oswalt \& H. E. Bond (Dordrecht: Springer), 445

García-Benito, R., Zibetti, S., Sánchez, S. F., et al. 2015, A\&A, 576, A135

Genzel, R., Tacconi, L. J., Gracia-Carpio, J., et al. 2010, MNRAS, 407, 2091

Genzel, R., Tacconi, L. J., Lutz, D., et al. 2015, ApJ, 800, 20

Glover, S. C. O., \& Clark, P. C. 2012, MNRAS, 426, 377

Helfer, T. T., Thornley, M. D., Regan, M. W., et al. 2003, ApJS, 145, 259

Hunter, J. D. 2007, CSE, 9, 90

Jameson, K. E., Bolatto, A. D., Leroy, A. K., et al. 2016, ApJ, 825, 12

Jogee, S., Scoville, N., \& Kenney, J. D. P. 2005, ApJ, 630, 837

Jones, E., Oliphant, T., Peterson, P., et al. 2001, SciPy: Open Source Scientific Tools for Python, http://www.scipy.org/

Kauffmann, G., Heckman, T. M., Tremonti, C., et al. 2003, MNRAS, 346, 1055

Kelly, B. C. 2007, ApJ, 665, 1489

Kendall, M. G. 1938, Biometrika, 30, 81

Kennicutt, R. C., \& Evans, N. J. 2012, ARA\&A, 50, 531

Kennicutt, R. C., Jr. 1998a, ARA\&A, 36, 189

Kennicutt, R. C., Jr. 1998b, ApJ, 498, 541

Kennicutt, R. C., Jr., Calzetti, D., Walter, F., et al. 2007, ApJ, 671, 333

Kewley, L. J., \& Dopita, M. A. 2002, ApJS, 142, 35

Kim, C.-G., Kim, W.-T., \& Ostriker, E. C. 2011, ApJ, 743, 25

Kim, C.-G., Ostriker, E. C., \& Kim, W.-T. 2013, ApJ, 776, 1

Kroupa, P. 2001, MNRAS, 322, 231

Kruijssen, J. M. D., \& Longmore, S. N. 2014, MNRAS, 439, 3239

Krumholz, M. R., Dekel, A., \& McKee, C. F. 2012, ApJ, 745, 69

Krumholz, M. R., \& Kruijssen, J. M. D. 2015, MNRAS, 453, 739
Krumholz, M. R., Leroy, A. K., \& McKee, C. F. 2011, ApJ, 731, 25

Kuno, N., Sato, N., Nakanishi, H., et al. 2007, PASJ, 59, 117

Leroy, A., Bolatto, A., Stanimirovic, S., et al. 2007, ApJ, 658, 1027

Leroy, A. K., Bigiel, F., de Blok, W. J. G., et al. 2012, AJ, 144, 3

Leroy, A. K., Bolatto, A., Gordon, K., et al. 2011, ApJ, 737, 12

Leroy, A. K., Schinnerer, E., Hughes, A., et al. 2017, ApJ, 846, 71

Leroy, A. K., Walter, F., Bigiel, F., et al. 2009, AJ, 137, 4670

Leroy, A. K., Walter, F., Brinks, E., et al. 2008, AJ, 136, 2782

Leroy, A. K., Walter, F., Sandstrom, K., et al. 2013, AJ, 146, 19

Lilly, S. J., Carollo, C. M., Pipino, A., Renzini, A., \& Peng, Y. 2013, ApJ, 772,119

Liu, G., Koda, J., Calzetti, D., Fukuhara, M., \& Momose, R. 2011, ApJ, 735, 63

Madau, P., \& Dickinson, M. 2014, ARA\&A, 52, 415

Makarov, D., Prugniel, P., Terekhova, N., Courtois, H., \& Vauglin, I. 2014, A\&A, 570, A13

Marino, R. A., Rosales-Ortega, F. F., Sánchez, S. F., et al. 2013, A\&A, 559, A114

Martins, L. P., González Delgado, R. M., Leitherer, C., Cerviño, M., \& Hauschildt, P. 2005, MNRAS, 358, 49

McKee, C. F., \& Ostriker, E. C. 2007, ARA\&A, 45, 565

Méndez-Abreu, J., Ruiz-Lara, T., Sánchez-Menguiano, L., et al. 2017, A\&A, 598, A32

Oka, T., Hasegawa, T., Sato, F., et al. 2001, ApJ, 562, 348

Osterbrock, D. E. 1989, Astrophysics of Gaseous Nebulae and Active Galactic Nuclei (Mill Valley, CA: Univ. Science Books), 422

Ostriker, E. C., McKee, C. F., \& Leroy, A. K. 2010, ApJ, 721, 975

Ostriker, E. C., \& Shetty, R. 2011, ApJ, 731, 41

Pettini, M., \& Pagel, B. E. J. 2004, MNRAS, 348, L59

Rahman, N., Bolatto, A. D., Xue, R., et al. 2012, ApJ, 745, 183

Rosolowsky, E., \& Blitz, L. 2005, ApJ, 623, 826

Rosolowsky, E., \& Leroy, A. 2006, PASP, 118, 590

Saintonge, A., Kauffmann, G., Kramer, C., et al. 2011a, MNRAS, 415, 32

Saintonge, A., Kauffmann, G., Wang, J., et al. 2011b, MNRAS, 415, 61

Salpeter, E. E. 1955, ApJ, 121, 161

Sánchez, S. F., Kennicutt, R. C., Gil de Paz, A., et al. 2012, A\&A, 538, A8

Sánchez, S. F., Pérez, E., Sánchez-Blázquez, P., et al. 2016, RMxAA, 52,171

Sánchez, S. F., Rosales-Ortega, F. F., Iglesias-Páramo, J., et al. 2014, A\&A, 563, A49

Sánchez-Blázquez, P., Peletier, R. F., Jiménez-Vicente, J., et al. 2006, MNRAS, 371, 703

Sánchez-Menguiano, L., Sánchez, S. F., Pérez, I., et al. 2016, A\&A, 587, A70

Sandstrom, K. M., Leroy, A. K., Walter, F., et al. 2013, ApJ, 777, 5

Sault, R. J., Teuben, P. J., \& Wright, M. C. H. 1995, in ASP Conf. Ser. 77, Astronomical Data Analysis Software and Systems IV, ed. R. A. Shaw, H. E. Payne, \& J. J. E. Hayes (San Francisco, CA: ASP), 433

Schinnerer, E., Meidt, S. E., Pety, J., et al. 2013, ApJ, 779, 42

Schmidt, M. 1959, ApJ, 129, 243

Schruba, A., Leroy, A. K., Walter, F., et al. 2011, AJ, 142, 37

Schruba, A., Leroy, A. K., Walter, F., et al. 2012, AJ, 143, 138

Schruba, A., Leroy, A. K., Walter, F., Sandstrom, K., \& Rosolowsky, E. 2010, ApJ, 722, 1699

Silk, J. 1997, ApJ, 481, 703

Singh, R., van de Ven, G., Jahnke, K., et al. 2013, A\&A, 558, A43

Spergel, D. N., \& Blitz, L. 1992, Natur, 357, 665

Vazdekis, A., Sánchez-Blázquez, P., Falcón-Barroso, J., et al. 2010, MNRAS, 404, 1639

Walcher, C. J., Wisotzki, L., Bekeraité, S., et al. 2014, A\&A, 569, A1

Weisz, D. R., Johnson, B. D., Johnson, L. C., et al. 2012, ApJ, 744, 44

Wilson, C. D., Warren, B. E., Israel, F. P., et al. 2012, MNRAS, 424, 3050

Wong, T., \& Blitz, L. 2002, ApJ, 569, 157

Wong, T., Xue, R., Bolatto, A. D., et al. 2013, ApJL, 777, L4

Wright, E. L., Eisenhardt, P. R. M., Mainzer, A. K., et al. 2010, AJ, 140, 1868 